\title{
Steady and unsteady laminar flows of Newtonian and generalized Newtonian fluids in a planar T-junction
}

\author{
A. I. P. Miranda ${ }^{1, \ddagger}$, P. J. Oliveira ${ }^{2, \S}$ and F. T. Pinho ${ }^{3,4, *, \dagger}$ \\ ${ }^{1}$ Departamento de Matemática, Universidade da Beira Interior, Rua Marquês D’Ávila e Bolama, \\ 6201-001 Covilhã, Portugal \\ ${ }^{2}$ Departamento de Engenharia Electromecânica, Universidade da Beira Interior, Centro de Materiais Texteis \\ e Papeleiros, Rua Marquês D’Ávila e Bolama, 6201-001 Covilhã, Portugal \\ ${ }^{3}$ Centro de Estudos de Fenómenos de Transporte, Faculdade de Engenharia da Universidade do Porto, \\ Rua Dr. Roberto Frias s/n, 4200-465 Porto, Portugal \\ ${ }^{4}$ Universidade do Minho, Largo do Paço, 4704-553 Braga, Portugal
}

\begin{abstract}
SUMMARY
An investigation of laminar steady and unsteady flows in a two-dimensional T-junction was carried out for Newtonian and a non-Newtonian fluid analogue to blood. The flow conditions considered are of relevance to hemodynamical applications and the localization of coronary diseases, and the main objective was to quantify the accuracy of the predictions and to provide benchmark data that are missing for this prototypical geometry. Under steady flow, calculations were performed for a wide range of Reynolds numbers and extraction flow rate ratios, and accurate data for the recirculation sizes were obtained and are tabulated. The two recirculation zones increased with Reynolds number, but the behaviour was non-monotonic with the flow rate ratio. For the pulsating flows a periodic instability was found, which manifests itself by the breakdown of the main vortex into two pieces and the subsequent advection of one of them, while the secondary vortex in the main duct was absent for a sixth of the oscillating period. Shear stress maxima were found on the walls opposite the recirculations, where the main fluid streams impinge onto the walls. For the blood analogue fluid, the recirculations were found to be $10 \%$ longer but also short lived than the corresponding Newtonian eddies, and the wall shear stresses are also significantly different especially in the branch duct. Copyright (C) 2007 John Wiley \& Sons, Ltd.
\end{abstract}

Received 29 May 2007; Revised 2 August 2007; Accepted 29 August 2007

KEY WORDS: T-junction; Newtonian; instability; blood analogue; Carreau-Yasuda model; twodimensional

\footnotetext{
*Correspondence to: F. T. Pinho, Centro de Estudos de Fenómenos de Transporte, Faculdade de Engenharia da Universidade do Porto, Rua Dr. Roberto Frias s/n, 4200-465 Porto, Portugal.

${ }^{\dagger}$ E-mail: fpinho@fe.up.pt

${ }^{\ddagger}$ E-mail: amiranda@mat.ubi.pt

§E-mail: pjpo@ubi.pt
}

Contract/grant sponsor: Fundação para a Ciência e Tecnologia; contract/grant number: PBICT/C/QUI/1980/95

Contract/grant sponsor: FEDER; contract/grant number: POCTI/EME/48665/2002 


\section{INTRODUCTION}

The numerical investigation of blood flows in veins and arteries is essential for a better understanding of the relationship between hemodynamics and cardiovascular diseases, a major cause of death in developed countries [1-5]. This continues to motivate research in this field, and there is today a drive to efficiently combine engineering and medicine tools [6]. In this context, an important flow geometry is the flow in bifurcating T-junctions [7].

In a large number of investigations on blood flow, the rheology is simplified and it is assumed that blood has a linear stress/strain relationship [5,8-11]. This simplification may be acceptable to a first approximation, especially in large blood vessels under steady conditions, but a more realistic fluid description is required for more accurate fluid dynamics. Indeed, it is well known that blood exhibits a complex rheology, behaving in a non-linear fashion, and this is even more evident whenever flows are unsteady and complex, such as in recirculating unsteady flows.

Accurate numerical investigations of complex three-dimensional unsteady flows require the simultaneous application of, at least, second-order discretization schemes applied on sufficiently refined meshes, and an accurate representation of fluid rheology. To verify that codes and numerical methods comply with these conditions, they are used first to calculate benchmark flows, such as the steady laminar lid-driven cavity flow for Newtonian fluids [12], the steady laminar flow in a 4:1 sudden contraction, or confined flows around cylinders with viscoelastic fluids [13,14]. However, there is a lack of benchmark data sets for flows of relevance to hemodynamical applications, especially flows involving unsteadiness, a state of things that this work aims to reduce. Specifically, this paper investigates steady and pulsating flows of Newtonian and, to a lesser extent, of generalized Newtonian fluids (GNF) through a planar $90^{\circ} \mathrm{T}$-junction, which is an adequate prototype of complex three-dimensional flows of biofluids in junctions and bifurcations. The aim is two-fold: to investigate the degree of space and time refinement required to obtain very accurate results, and to obtain values of quantities such as the size of the separated flow regions and shear stresses on walls at relevant Reynolds numbers and flow rate ratios for fluids possessing an adequate fluid rheology.

Blood is a suspension of platelets and cells in plasma, which is a mixture of water and long molecules such as proteins and enzymes. The cells are deformable and can also agglomerate into the so-called rouleaux which orientate and deform anisotropically $[15,16]$. Cell agglomeration is determined by the shear rate as well as by the concentration of macromolecules in the plasma (fibrinogen), amongst other things. As a consequence of this complexity, blood is a thixotropic fluid [17], possessing an yield stress [18], and exhibiting elasticity [19] and a shear-thinning viscosity [20], as is well recognized by some authors [5]. Still, it is often considered by many researchers that flow characteristics of blood can be well modelled by a Newtonian equation when it occurs in large blood vessels and under steady flow conditions [1,21-23]. However even in large blood vessels, flow unsteadiness leads to regions of low deformation rate where the viscoelasticity of blood becomes especially relevant and affecting the stress field. This has been shown by Vlastos et al. [19], who measured blood rheology under combined oscillatory and steady shear, and recently by Bachmann et al. [24], who measured the flows of Newtonian and non-Newtonian blood analogs in a pediatric ventricle with handmade ball and cage valves at normal physiologic conditions and showing the dramatic differences in wall shear stresses. Therefore, the assumption of Newtonian behaviour of blood should be taken with caution and there is the need to investigate in more detail the dynamics of non-Newtonian fluids under unsteady flow conditions of relevance to hemodynamics. Such investigation should address first the effects of variable viscosity coupled 
with unsteadiness, as in this contribution, followed by an assessment of the role of viscoelasticity, which will be addressed in a forthcoming paper. As a matter of fact, recent research [25] has shown that even in the absence of elasticity the shear thinning of viscosity is enough to complicate matters and to ensure that complete dynamic similarity between blood and a Newtonian model fluid becomes an impossible task.

Studies on bifurcating flows were done as early as the 1920s by Vogel [26], who quantified pressure losses in a T-junction. The widespread use of T-junctions in piping networks and the corresponding pressure losses have been a major motivation for research on steady T-junction flows, as recently reviewed by Costa et al. [27]. In living organisms bifurcating flows have characteristics of their own, such as flow unsteadiness, non-linear rheology, specific flow geometries (the angle of bifurcation is usually less than $90^{\circ}$ and the vessels have taper), which justify the following more focused review.

The availability of optical diagnostic techniques from the 1970s onwards has paved the way to detailed investigations of liquid flows in complex geometries of relevance to hemodynamics. Karino et al. [28] visualized vortex shedding from the corner of a bifurcation and its relationship with the curvature of the edge of the T-junction. Liepsch et al. [29] used laser-Doppler anemometry (LDA) to measure the steady laminar flow of water in a bifurcation formed by rectangular ducts having an aspect ratio of 8:1 and performed the corresponding numerical calculations assuming two-dimensional flow and using first-order discretization schemes for convection. The numerical and experimental results agreed qualitatively, showing the existence of a main recirculation in the branch duct and a secondary eddy starting inside the junction at the wall opposite the branch duct and going into the main duct.

Numerical calculations of steady flow by Khodadadi et al. [30], using a finite volume method, were carried out in the same two-dimensional geometry of Liepsch et al. [29] and included heat transfer, but they relied on the hybrid scheme for convection, another first-order discretization scheme. At high Reynolds numbers and high values of the flow rate ratio, a third recirculation region was found in the downstream main duct. This research was later extended by Khodadadi et al. [31], who have carried out simulations and experiments on pulsatile flow using LDA. Their unsteady flow calculations relied as well on first-order discretization schemes for both the space and time discretizations that, as we know today, are not accurate due to excessive numerical diffusion especially in the recirculation regions where mis-alignment between velocity vectors and mesh is large. However, the experimental data set of Khodadadi et al. [31] is very comprehensive and provides local velocity measurements that are useful to check the accuracy of numerical methods, as we have done here. Subsequently, Khodadadi [32] presented further experimental and numerical data, namely the shear stress along the walls, which is of relevance to establish the relationship between cardiovascular disease, flow recirculation and the magnitude of shear stresses. In these investigations by Khodadadi et al., discrepancies between predictions and experimental data were found in the main recirculation and were partly attributed to three-dimensional effects in the experiments, but especially to excessive numerical diffusion which was quantified in Khodadadi et al. [30].

The same two-dimensional bifurcation was used again by Moravec and Liepsch [33], who performed further experiments with LDA using Newtonian and non-Newtonian fluids. Subsequently, Liepsch and Moravec [34] investigated pulsating flows in rigid and distensible arteries and in the latter case they found that the wall elasticity reduced the size of the secondary recirculation. Rindt and Stenehoven [35] investigated numerically the unsteady flow in a rigid carotid artery, but have not validated their numerical predictions. Also of relevance to hemodynamics, the investigation 
of Ravensbergen et al. [9] has concentrated on junction flow, rather than the bifurcating flow of interest in the present work.

What is clear from this review is the lack of proper validation and assessment of numerical accuracy in works on steady and pulsatile flows of Newtonian as well as non-Newtonian fluids through T-junctions, and that constitutes the objective of the present investigation. In this first contribution, the emphasis is on quantitative results for the Newtonian two-dimensional case, of benchmark quality, before embarking upon more complicated three-dimensional and non-Newtonian calculations. Nevertheless, some GNF simulations are also presented, enough to show how misleading the assumption of constant viscosity can be. In a second paper we shall deal with the GNF case in more depth. The remaining part of this paper is organized as follows: the governing equations and the numerical methods used are presented in Section 2. The results and discussion section is organized in four parts: the first part starts with the presentation of the flow geometry, computational domain and meshes used, then it discusses issues of mesh convergence and space discretization and finally it presents accurate predictions of steady T-junction laminar flow of Newtonian fluids as a function of the flow rate ratio and Reynolds number. The second part assesses the time accuracy of unsteady flow calculations by comparing predictions of unsteady laminar channel flow of Newtonian fluids with the corresponding analytical solution. Results are presented in part three for the unsteady flow in a diverging laminar $90^{\circ} \mathrm{T}$-junction with a Newtonian fluid and finally, in part 4 , for a Generalized Newtonian fluid. In all cases, the fluids selected have properties that closely follow those of blood. The paper ends with a summary of the main conclusions.

\section{GOVERNING EQUATIONS AND NUMERICAL METHOD}

The governing equations were those of mass conservation and momentum balance for isothermal incompressible time-dependent flows, expressed here in index notation. They are

$$
\begin{gathered}
\frac{\partial u_{i}}{\partial x_{i}}=0 \\
\rho \frac{\partial u_{i}}{\partial t}+\rho \frac{\partial\left(u_{i} u_{j}\right)}{\partial x_{j}}=-\frac{\partial p}{\partial x_{i}}+\frac{\partial \tau_{i j}}{\partial x_{j}}
\end{gathered}
$$

where $\rho$ is the fluid density, $u_{i}$ is the component of the velocity vector along coordinate $x_{i}, p$ is the pressure and $\tau_{i j}$ is the extra stress tensor. The extra stress tensor is given by the rheological constitutive equation for the GNF model:

$$
\tau_{i j}=\eta(\dot{\gamma}) \dot{\gamma}_{i j}
$$

where the rate of deformation tensor $\left(\dot{\gamma}_{i j}\right)$ is defined as $\dot{\gamma}_{i j} \equiv u_{i, j}+u_{j, i}$ and the scalar $\dot{\gamma}$ is related to the second invariant of $\dot{\gamma}_{i j}$, namely

$$
\dot{\gamma}=\sqrt{\frac{1}{2} \dot{\gamma}_{k l} \dot{\gamma}_{k l}}
$$

The viscosity function here adopted was the five-parameter Carreau-Yasuda (CY) model:

$$
\eta=\eta_{\infty}+\left(\eta_{0}-\eta_{\infty}\right)\left[1+(\lambda \dot{\gamma})^{a}\right]^{(n-1) / a}
$$


where $\eta_{0}$ is the zero-shear-rate viscosity, $\eta_{\infty}$ is the infinite-shear-rate viscosity, $\lambda$ is a time constant, $n$ is the power law region exponent and $a$ is a non-dimensional parameter related to the smoothness of the transition between the zero-shear-rate constant viscosity plateau and the power law viscosity region. A common value of $a$ is 2 , which provides a good fitting to experimental data of many fluids, in which case the model is simply called Carreau model. Setting $n=1$, the fluid becomes Newtonian with viscosity coefficient $\eta=\eta_{0}$.

Equations (1)-(5) were solved numerically with a finite volume method identical to that used in previous works by the authors [14,36,37]; hence, only a brief account is given here emphasizing the discretization of the inertial term. The momentum and continuity equations were initially written in a non-orthogonal coordinate system, but keeping the Cartesian velocity and stress tensor components as the main dependent variables. These differential equations were then transformed into algebraic equations by means of a finite volume discretization on a collocated, non-orthogonal mesh, as described by Oliveira et al. [36]. The space discretization of all terms relied on high-order accurate schemes: central differences for the diffusive terms and the CUBISTA scheme of Alves et al. [38] for the convective terms, which are formally of second and third order on uniform meshes, respectively. For the temporal discretization of the unsteady term of the momentum equation, the three time level scheme described by Oliveira [37] was used, which is also formally of second-order accuracy.

The discretized momentum equation for the velocity component $u_{i}$ (of vector $\mathbf{u}$ ) at a general cell $P$ is given by

$$
a_{P} \mathbf{u}_{P}^{*}=\sum_{F} a_{F} \mathbf{u}_{F}^{*}+\left\{-\nabla p^{*}+\nabla \cdot \tau^{*}+S_{u}^{\mathrm{HOS}}+\frac{\rho V_{P}}{\Delta t}\left(2 \mathbf{u}_{P}^{(n)}-0.5 \mathbf{u}_{P}^{(n-1)}\right)\right\}
$$

where the central coefficient $a_{P}=1.5 \rho V_{P} / \Delta t+\sum_{F} a_{F}$, with $V_{P}$ the volume of the cell centred at $P, \Delta t$ the time step and the neighbour coefficients $a_{F}=D_{f}+F_{f}$ having diffusive $\left(D_{f}\right)$ and convective contributions $\left(F_{f}\right)$. In the coefficient $a_{F}$ the convective contributions are of first order (upwind scheme, UDS), since the high-resolution CUBISTA scheme is implemented via deferred correction, see Oliveira et al. [36] and Alves et al. [38], and the difference between the CUBISTA and the first-order convective fluxes is dealt with explicitly in $S_{u}^{\mathrm{HOS}}$. Computation of convective fluxes of momentum was also carried out using the second-order linear upwind (LUDS) and central difference (CDS) schemes, which were also implemented into the code via the deferred correction approach, in which cases $S_{u}^{\mathrm{HOS}}$ contains the difference between the fluxes calculated with the LUDS/CDS scheme and the UDS.

The velocity field $\mathbf{u}^{*}$ obtained from the implicit solution of Equation (6) is still imperfect and will not satisfy continuity at the next iteration level/time step (superscripts $* *$ and $(n+1)$ ),

$$
\nabla \cdot \mathbf{u}^{* *}=0
$$

therefore, the intermediate velocity $\mathbf{u}^{*}$ and pressure $p^{*}$ need to be corrected. The corrected velocity $\left(\mathbf{u}^{* *}\right)$ is determined from a factored form of the momentum equation:

$$
\left(1.5 \frac{\rho V_{P}}{\Delta t}\right) \mathbf{u}_{P}^{* *}+\sum_{F} a_{F} \mathbf{u}_{P}^{*}=\sum_{F} a_{F} \mathbf{u}_{F}^{*}-\nabla p^{* *}+\nabla \cdot \tau^{*}+S_{u}^{\mathrm{HOS}}+\frac{\rho V_{P}}{\Delta t}\left(2 \mathbf{u}_{P}^{(n)}-0.5 \mathbf{u}_{P}^{(n-1)}\right)
$$

where the inertial and the pressure gradient terms only have been updated to the new iteration level $(* *)$. 
The pressure correction is $p^{\prime}=p^{* *}-p^{*}$, where $p^{*}$ denotes the existing or previous iteration level, and it is calculated with a Poisson-like pressure correction equation derived by subtracting Equation (6) from Equation (8), while imposing the continuity condition (Equation (7)). The equation for $p^{\prime}$ is

$$
a_{P}^{p} p_{P}^{\prime}=\sum_{F} a_{F}^{p} p_{F}^{\prime}-\nabla \cdot \mathbf{u}^{*}
$$

with $a_{P}^{p}=\sum_{F} a_{F}^{p}, a_{F}^{p}=A_{f}^{2} /\left(1.5 \rho V_{P} / \Delta t\right)$ and $A_{f}$ denoting cell-face areas.

In this work the stress is an explicit function of the deformation rate tensor, but was solved separately since the calculations were carried out using a viscoelastic code, where the stress is calculated by a differential constitutive equation. Here,

$$
\tau_{i j}^{*}=\eta\left(\dot{\gamma}^{* *}\right) \dot{\gamma}_{i j}^{* *}
$$

where the superscript $* *$ applies to the newly calculated, continuity-satisfying velocity from Equation (8). When starting the next time step, after converging the iterations within the present time, we do $\tau^{n+1}=\tau^{*}, \mathbf{u}^{n+1}=\mathbf{u}^{* *}$ and $p^{n+1}=p^{* *}$.

The linearized sets of algebraic equations to be solved (Equations (6) and (9)) are of the form

$$
a_{P} \phi_{P}=\sum_{F} a_{F} \phi_{F}+b
$$

where the summation for index $F$ is over the six cell neighbours of cell $P$. The systems of equations are solved iteratively with the conjugate gradient method preconditioned with an incomplete LU decomposition for the symmetric $p^{\prime}$ equation, and the bi-conjugate gradient method for the velocity components.

There are two levels of iteration in this time-dependent algorithm. An inner set of iterations inside the solvers is pursued until the initial residuals on entering the solver decay by two orders of magnitude, and an outer iteration level, inside a time step $\delta t$, because the momentum equation (6) depends on the stress field, the stress depends on the velocity field, the factored momentum equation (8) is only approximate and there are explicit non-linearities in the advective terms of momentum and in the viscosity function. These outer iterations are repeated through Equations (6)(10) until $\tau^{*}, p^{* *}$ and $\mathbf{u}^{* *}$ do not change. This is controlled by the normalized $L_{1}$ residuals of the equations, which are required to be below a tolerance of $10^{-4}$, and only then the calculation proceeds to the next time level.

There are three types of boundary conditions relevant to the present flow problems: inlets, outlets and solid walls. At the inlet, the streamwise velocity component and the shear stress component are prescribed, based on available analytical solutions. Typically, the velocity follows a parabolic shape for the steady flows and the Womersley solution for the pulsating flows. At the walls the no-slip condition is applied directly, as a Dirichelet condition, and the shear stress is calculated from the local velocity distribution. Finally, at the two outlets of the T-junction one can either prescribe the pressure, and the flow split is an outcome of the calculation, or prescribe the flow rates in each of the outlets. We have decided for the latter, since we wish to vary the flow rate ratio $\beta$ as a controlling, given parameter. It is important to notice that, when the flow rates are prescribed, the problem is not well posed if the flow rates are not fixed separately for each outlet. In practice, for the implementation of this outlet boundary conditions, the $u^{*}$ obtained after solution of the momentum equations (6) is linearly extrapolated to the two outlet planes, and then a bulk velocity correction is applied separately to the outlet along the main duct, so that the outlet flow 
rate here is exactly $Q_{2}=(1-\beta) Q_{1}\left(Q_{1}\right.$ is imposed at inlet), and the outlet flow rate along the side branch is $Q_{3}=\beta Q_{1}$. In this way, overall mass conservation is satisfied exactly before solving the pressure equation (9), a necessary condition for the existence of a solution for this equation.

Following the methodology of Roache [39], the code implementing this numerical method has been verified and validated several times with Newtonian and non-Newtonian inelastic fluids. Two such cases are the detailed steady annular flow predictions of Escudier et al. [40, 41] against experimental data from several authors and including also extensive verifications using several meshes and Richardson extrapolation to the limit. Regarding the bifurcating flow geometry, Section 3.1.1 is entirely devoted to verification for steady flow, Section 3.2 verifies issues of time dependency and the validations against several sets of experimental data for steady flow and one unsteady analytical solution are presented in Sections 3.1.2 and 3.2, respectively.

\section{RESULTS AND DISCUSSION}

Here we present results for steady and unsteady laminar diverging flow in a two-dimensional $90^{\circ} \mathrm{T}$ junction, but prior to the parametric investigation an assessment is made of numerical uncertainties by studies of the effects of mesh refinement, numerical interpolation scheme and time step upon the numerical results. The effects of mesh refinement and the results for the steady flow in the $90^{\circ}$ T-junction are dealt with in Section 3.1, the effects of time discretization and the corresponding uncertainties are dealt with in Section 3.2 with reference to the analytical solution for periodic flow in a channel and then Section 3.3 presents results for the Newtonian unsteady flow in the diverging T-junction. Finally, Section 3.4 deals with the unsteady diverging T-junction flow for a non-Newtonian Carreau fluid analogue to blood.

Hence, there are two geometries in this section: the channel flow in Section 3.2 and the $90^{\circ}$ T-junction everywhere else. The description of the channel flow geometry is very specific and is left for Section 3.2 where the time-dependent procedure is verified and validated.

The T-junction flow geometry is the same as that of Khodadadi et al. [30, 31] and Khodadadi [32] and is schematically represented in Figure 1. The origin of the coordinate system is at the centre of the bifurcation. The inlet duct is denoted with subscript 1 , and the main and branch outlet ducts are denoted by subscripts 2 and 3, respectively. All ducts have the same width, $H$. The flow rate ratio is defined as $\beta \equiv Q_{3} / Q_{1}$, where $Q_{1}$ and $Q_{3}$ are the inlet duct and branch duct flow rates per unit span, respectively. In each duct the bulk velocity is defined as the ratio between the corresponding flow rate and duct width, as in $\bar{u}_{1}=Q_{1} / H$. The main recirculation is in the branch duct starting at $y=y_{s}$ and ending at $y=y_{r}$, thus defining a normalized recirculation length of $Y_{R}=\left(y_{r}-y_{s}\right) / H$. This nomenclature is adapted for the secondary recirculation found in the main duct and aligned with the $x$-direction with the necessary adaptations leading to $X_{R}=\left(x_{r}-x_{s}\right) / H$.

\subsection{Steady laminar diverging flow of Newtonian fluids in a $90^{\circ}$ T-junction}

3.1.1. Effects of mesh refinement and discretization scheme. The laminar flow under investigation here is the steady two-dimensional diverging flow of Newtonian fluids in the $90^{\circ} \mathrm{T}$-junction. Previous works from the literature on this flow are summarized in Table I. The numerical investigations relied on rather coarse meshes and most calculations were performed using first-order discretization schemes for advection known to be excessively diffusive on such coarse grids. The exception seems to be the more recent work of Neary and Sotiropoulos [45], which apparently used 


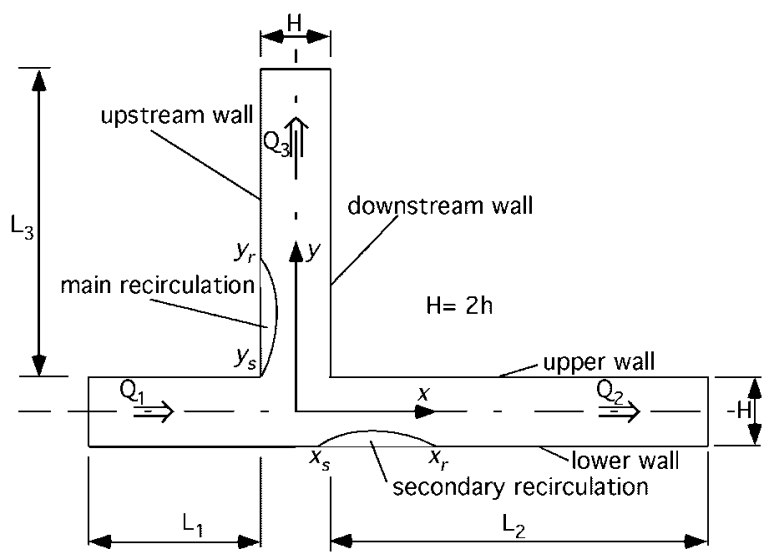

Figure 1. Geometry of the bifurcation and coordinate system.

Table I. Previous numerical studies in two-dimensional and three-dimensional diverging T-junction flows.

\begin{tabular}{|c|c|c|c|c|c|c|}
\hline Reference & Method & Scheme & Mesh & $N C$ & $N$ & 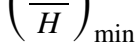 \\
\hline Vlachos [42] & FVM, 2D & Hybrid & $44 \times 34$ & $\approx 992$ & 16 & 0.0625 \\
\hline al. [29] & Exp. and numerical & $\mathrm{H}$ & 44 & $\approx 992$ & 16 & 0.0625 \\
\hline Khodadadi et al. [30] & FVM, 2D & Hybrid & $60 \times 60$ & - & - & - \\
\hline al. [31] & FVM, 2D & Hybrid & $36 \times 35$ & $\approx 880$ & $\approx 16$ & 0.0625 \\
\hline al. [43] & FEM, 2D & $\mathrm{u}$-quadratic, $\mathrm{p}$-linear & Triangles & 1470 & 7 & 0.14 \\
\hline Collins and $\mathrm{Xu}$ [44] & FVM, 2D, 3D & Vector upwind & - & 840 & 10 & 0.1 \\
\hline Khodadadi [32] & FVM, 2D & & $36 \times 3$ & $\approx 880$ & $\approx 16$ & 0.0625 \\
\hline iropoulos [45] & FDM, 2 & LU & $100 \times 80$ & 3200 & 20 & 0.02 \\
\hline This work & FVM, 2D & CUBISTA, CDS, LUDS & $360 \times 280$ & 44800 & 80 & 0.0125 \\
\hline
\end{tabular}

$N C$, Total number of cells within flow domain; $N$, the number of cells in transverse direction (wall-to-wall).

${ }^{*}$ It is not absolutely clear that LUDS was used here.

the second-order LUDS and a finer mesh containing 20 cells from wall to wall (with a normalized minimum spacing of 0.02). In our work, three consecutively refined meshes (M1-M3) were used initially to investigate mesh convergence, the details of which are given in Table II; our finest mesh had 80 cells, uniformly distributed inside the junction region from wall to wall (block 2, see below), giving a normalized cell size of 0.0125 . Later, namely for the results in Sections 3.3 and 3.4, we have prepared mesh M4, patterned after M2 but with a non-uniform distribution of the 40 cells across the channels enabling a minimum mesh spacing of 0.01 .

The first set of calculations is directly comparable with those of Liepsch et al. [29] and pertains to a Reynolds number of $248\left(R e \equiv \rho \bar{u}_{1} H / \eta_{0}\right)$ and $\beta=0.44 . \eta_{0}$ refers to the viscosity of Newtonian fluids and the zero-shear-rate viscosity for non-Newtonian fluids. Note that the works in Table I define the Reynolds number differently and the results may not be comparable; for some authors there is some uncertainty regarding their definition of Reynolds number.

As mentioned above, the inlet condition was set as fully developed with the corresponding velocity profile having a parabolic shape. Downstream the bifurcation the flow becomes fully 
Table II. Some characteristics of the computational meshes.

\begin{tabular}{|c|c|c|c|c|c|c|}
\hline Mesh & $N C$ & $N$ & $f_{x_{1}}$ & $f_{x_{2}}$ & $f_{y_{3}}$ & $\left(\frac{\Delta x}{H}\right)_{\min }$ \\
\hline M1 & 2800 & $20 \mathrm{U}$ & 0.9074 & 1.05385 & 1.04827 & 0.05 \\
\hline M2 & 11200 & $40 \mathrm{U}$ & 0.9526 & 1.02657 & 1.02385 & 0.025 \\
\hline M3 & 44800 & $80 \mathrm{U}$ & 0.9760 & 1.01320 & 1.01185 & 0.0125 \\
\hline M4 & 14000 & $40 \mathrm{NU}$ & 0.9425 & 1.02554 & 1.02750 & 0.01 \\
\hline M5 & 14800 & $40 \mathrm{UL}$ & 0.9526 & 1.02657 & 1.02385 & 0.025 \\
\hline
\end{tabular}

U, Uniform; NU, Non-uniform; L, longer arms. M1, M2, M3: $L_{1}=3 H, L_{2}=10 H, L_{3}=12 H ; \mathrm{M} 5: L_{2}=22 H$, $L_{3}=20 H . f_{y_{3}} \equiv \Delta y_{i+1} / \Delta y_{i}$ in block $3 ; i+1$ and $i$ indicate consecutive cells.

developed, provided the branch and main ducts are sufficiently long. According to Shah and London [46] the pipe length required to develop a Newtonian flow is given by

$$
\frac{L}{H}=\frac{0.315}{0.068 R e+1}+0.044 R e
$$

with our definition of Reynolds number, leading to values of $L_{2} / H$ and $L_{3} / H$ of 6.2 and 4.9, respectively. To guarantee that the outlet boundary conditions did not affect the calculations, the computational domain was set to have longer lengths of $L_{2} / H=12$ and $L_{3} / H=10$, whereas for the inlet duct $L_{1} / H=3$ was used. For the results of Section 3.1.2, where larger values of $R e$ and $\beta$ were considered, these duct lengths were further extended by $10 H$, giving a main duct length of $L_{2}=22 \mathrm{H}$ and branch duct length of $L_{3}=20 \mathrm{H}$ (in mesh M5 of Table II, based on M2). In addition, a few runs were made on a mesh with $L_{2} / H=42$ and $L_{3} / H=40$ to conform with Equation (12) for the highest $R e$ and $\beta$, but the results in terms of vortex size were the same as on mesh M5.

The meshes are semi-structured and were made from 4 blocks of structured cells: block 1 at the inlet duct, block 2 at the main outlet duct, block 3 for the branch duct and block 4 corresponded to the square junction of side $H$. The cells within block 4 were squares of uniform size, in block 1 they concentrated toward the junction with a concentration factor $f_{x 1}$ and in blocks 2 and 3 they expanded away from the junction with expansion factors $f_{x 2}$ and $f_{y 3}$, respectively. The concentration factor $f_{x 1}$ is the ratio of consecutive cell lengths in the $x$-direction in block 1, i.e. $f_{x_{1}} \equiv \Delta x_{i+1} / \Delta x_{i}$, the other factors being defined similarly after the necessary adaptations. The details of the meshes are given in Table II and Figure 2 gives an idea of mesh M2 near the bifurcation and the blocks used to generate it.

We performed calculations for the experimental case of Liepsch et al. [29] with meshes M2 and M3 and using CDS and LUDS schemes. The computed velocity profiles compared well with the experimental data, where these data were available, and Figure 3 shows some selected comparison of profiles in the main duct and in the side branch. Here predictions with the UDS are included for the purpose of highlighting the kind of errors it may generate. In contrast, the numerical results obtained with CDS and LUDS are indistinguishable. In other comparisons not shown, obtained with meshes M2 and M3, the results are also indistinguishable in these same planes but may differ in some other planes. An assessment of the mesh effect on results can be grasped in Table III, which lists values of the lengths of the two separation bubbles, $X_{R}$ and $Y_{R}$. The use of Richardson extrapolation to the limit provides very accurate values of the two quantities, theoretically of fourth order (since CDS is formally of second order in uniform meshes). The uncertainty in the results is of the order of $0.1 \%$ for mesh M3 and of $0.2 \%$ for mesh M2. These results also show that the 


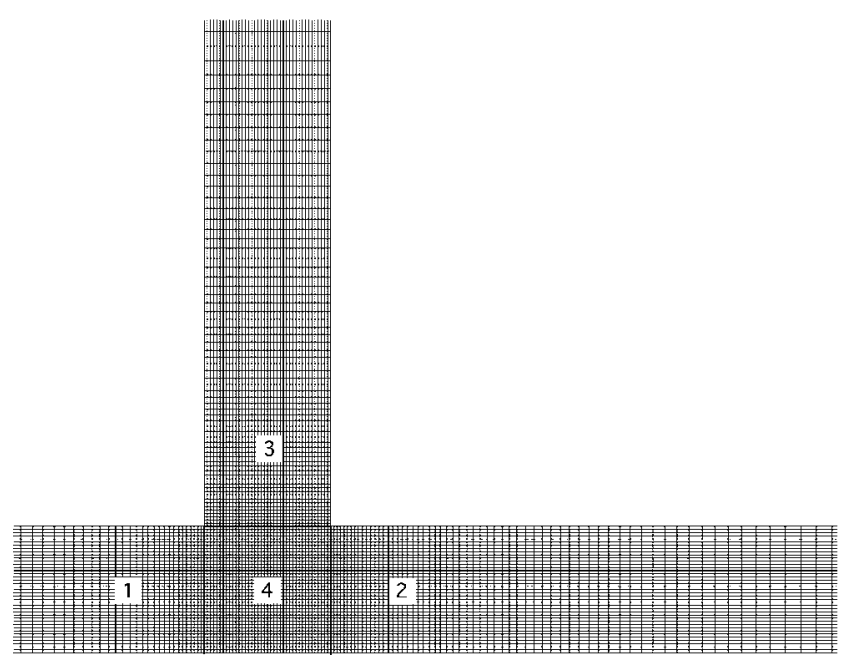

Figure 2. Detail of computational domain and mesh M2.
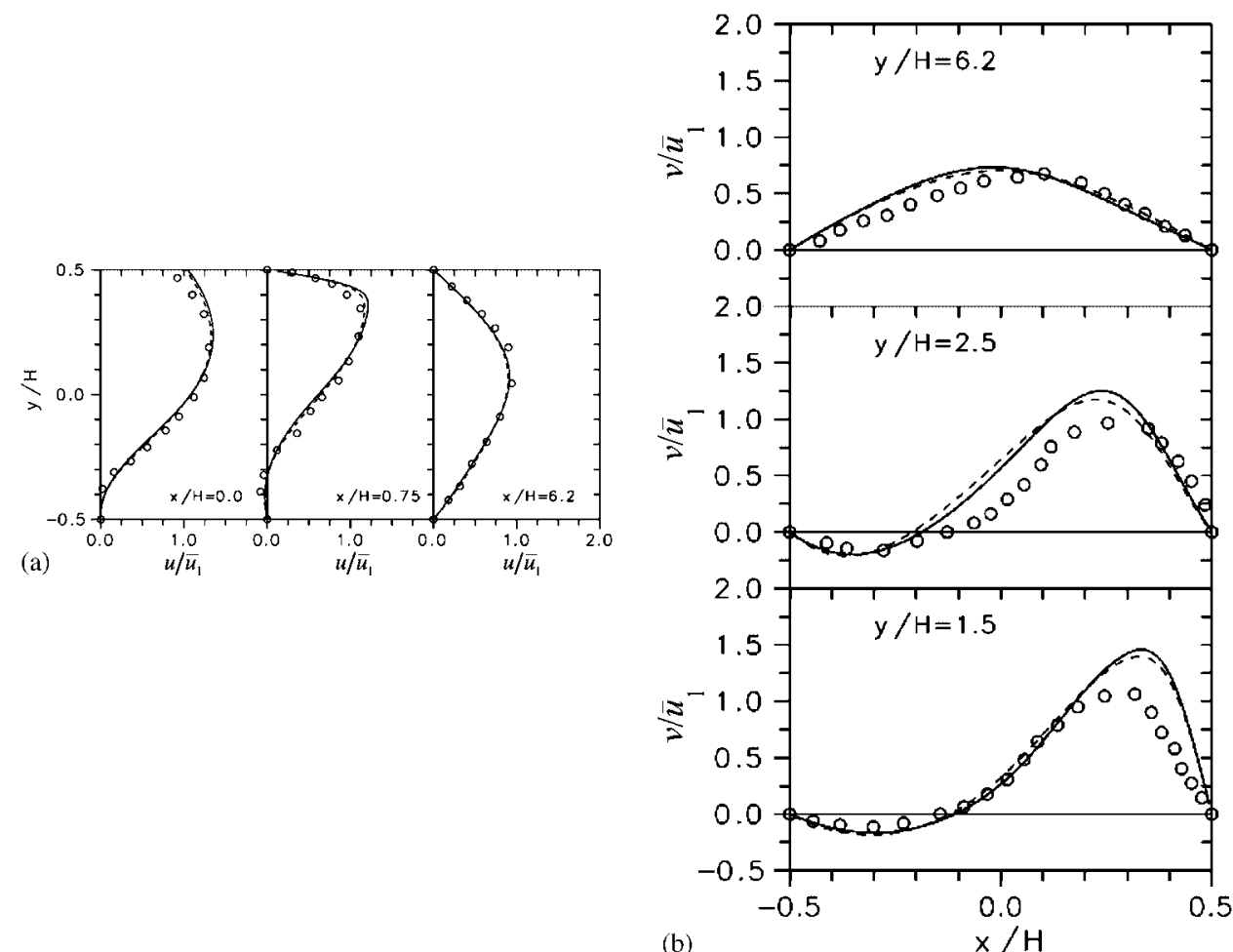

Figure 3. Comparison between experimental data of Liepsch et al. [29] ( $R e=248$ and $\beta=0.44)$ and predictions using mesh M3 for various differencing schemes (full line, CDS; long dash, LUDS; short dash, UDS): (a) main outlet duct and (b) branch duct. 


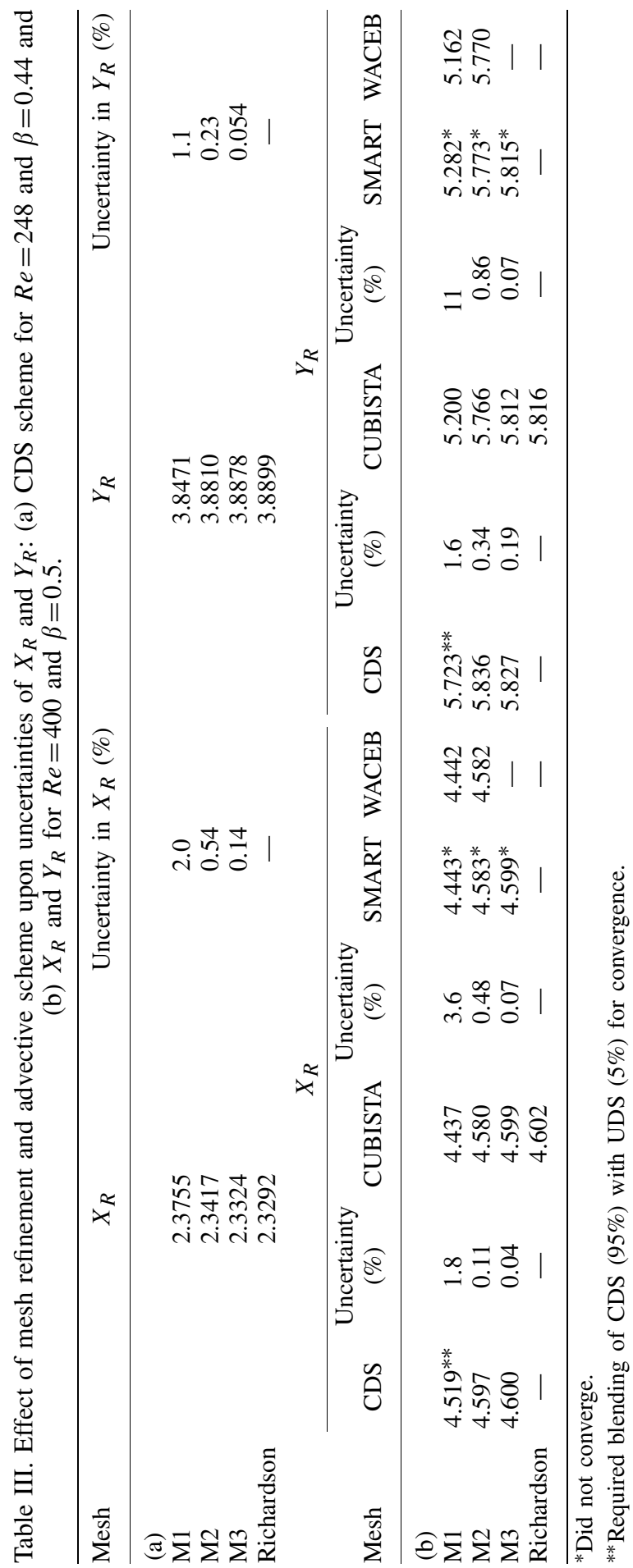


order of convergence for the CDS scheme was between 1.9 and 2.3 for $X_{R}$ and $Y_{R}$, respectively, i.e. close to the theoretical value.

Table III also contains $X_{R}$ and $Y_{R}$ data for $R e=400$ and $\beta=0.5$ using CDS and CUBISTA, as well as the high-resolution schemes SMART of Gaskell and Lau [47] and WACEB of Song et al. [48] used to help confirm the results obtained with the CUBISTA scheme. For $X_{R}$ the predictions of CUBISTA and CDS already agree for mesh M3 to within $0.1 \%$, whereas for $Y_{R}$ CDS is taking longer than CUBISTA to converge to a mesh-independent value. The data obtained with the CUBISTA scheme are converging monotonically to a constant value, but the simulation with CDS for mesh M1 is not yet in the monotonic convergence region as seen in Table III(b). In fact, for this calculation it was actually necessary to blend 5\% of UDS (with 95\% CDS) in order to eliminate some numerical stability problems. Owing to the differences between the predictions of CDS and CUBISTA, similar calculations were carried out with the WACEB and SMART high-resolution schemes, which are also based on the QUICK scheme and consequently are also formally of third order in uniform meshes. These simulations with SMART and WACEB confirmed the predictions of CUBISTA, as is also shown in Table III. On meshes M2 and M3 the results of CUBISTA were not as close to the extrapolated data as those of CDS, but in all cases CUBISTA was more robust than CDS from the point of view of iterative convergence.

It is clear from the above that the use of mesh M2 in combination with CUBISTA provides very robust iterative convergence in combination with sufficiently accurate values; in particular, for the predictions of $X_{R}$ and $Y_{R}$ the uncertainties are below $1 \%$ and therefore can be considered at this stage as benchmark data, a significant improvement over existing literature data for this flow, which were obtained 10-15 years ago with coarser meshes and using low-order differencing schemes of the upwind type.

3.1.2. Parametric investigation on the effects of flow rate ratio and Reynolds number. We give now and discuss the main quantitative results in terms of vortex size data for the steady flow in the T-junction. The flow rate ratio $(\beta)$ and Reynolds number were varied between 10 and $90 \%$ and 50 and 1000, respectively, and the corresponding results are listed in Table IV and compared with the predicted results of Khodadadi et al. [30] in Figure 4.

As mentioned with regard to Table I, predictions in the literature relied on rather coarse meshes and first-order schemes, and consequently are not sufficiently accurate and suffer from excessive numerical diffusion. This is made clear in Figure 4, where the results of Khodadadi et al. [30] are compared with our own predictions on mesh M5 (similar to M2, but with longer outlet ducts) for several values of $\beta$. It is worth mentioning that Khodadadi et al. [30] have attempted to quantify the numerical diffusivity for $\beta=0.5$ and found values in excess of the molecular diffusion at high Reynolds number flows, especially in the branch duct and outside the main recirculation, i.e. in regions of large velocity and misalignment between the grid and the velocity vectors. Indeed, at low Reynolds numbers the agreement for $X_{R}$ is good, but only fair for $Y_{R}$, because the flow in the main duct is more aligned with the mesh, and hence has less numerical diffusion, than the flow in the initial part of the branch duct. The higher numerical diffusion in the branch leads to faster flow redevelopment and shorter recirculation lengths. Consequently, the discrepancies increase with Reynolds number, because the numerical diffusion contaminating Khodadadi's predictions, obtained with a first-order hybrid scheme, grows in magnitude with the cell Peclet number (or velocity vector magnitude) due to the misalignment between the mesh and the velocity vectors, whereas the magnitude of molecular diffusion diminishes. 
Table IV. Main and secondary recirculation lengths as a function of $\operatorname{Re}$ and $\beta$.

\begin{tabular}{rllll}
\hline$R e$ & $x_{S} / H$ & $x_{r} / H$ & $y_{S} / H$ & $y_{r} / H$ \\
\hline (a) $\beta=0.1$ & & & & \\
50 & 0 & 0 & 0.516 & 1.294 \\
100 & 0 & 0 & 0.510 & 1.549 \\
150 & 0 & 0 & 0.507 & 1.769 \\
200 & 0 & 0 & 0.506 & 1.964 \\
250 & 0 & 0 & 0.504 & 2.142 \\
300 & 0 & 0 & 0.504 & 2.307 \\
350 & 0 & 0 & 0.503 & 2.461 \\
400 & 0 & 0 & 0.502 & 2.603 \\
450 & 0 & 0 & 0.502 & 2.734 \\
500 & 0 & 0 & 0.502 & 2.859 \\
600 & 0 & 0 & 0.501 & 3.090 \\
700 & 0 & 0 & 0.501 & 3.301 \\
800 & 0 & 0 & 0.500 & 3.500 \\
900 & 0 & 0 & 0.500 & 3.691 \\
1000 & 0 & 0 & 0.500 & 3.875
\end{tabular}

(b) $\beta=0.3$

$\begin{array}{rllll}50 & 0 & 0 & 0.5247 & 1.452 \\ 100 & 0 & 0 & 0.5154 & 2.095 \\ 150 & 0 & 0 & 0.5115 & 2.668 \\ 200 & 0.452 & 0.865 & 0.5102 & 3.204 \\ 250 & 0.233 & 1.269 & 0.509 & 3.699 \\ 300 & 0.120 & 1.586 & 0.508 & 4.141 \\ 350 & 0.0401 & 1.884 & 0.508 & 4.520 \\ 400 & -0.0229 & 2.178 & 0.507 & 4.836 \\ 450 & -0.0751 & 2.472 & 0.507 & 5.108 \\ 500 & -0.120 & 2.769 & 0.507 & 5.349 \\ 600 & -0.194 & 3.374 & 0.506 & 5.784 \\ 700 & -0.254 & 3.992 & 0.506 & 6.175 \\ 800 & -0.304 & 4.622 & 0.506 & 6.524 \\ 900 & -0.346 & 5.260 & 0.506 & 6.826 \\ 1000 & -0.386 & 5.902 & 0.505 & 7.106\end{array}$

(c) $\beta=0.5$

$\begin{array}{rllll}50 & 0 & 0 & 0.532 & 1.538 \\ 100 & 0.217 & 1.001 & 0.523 & 2.400 \\ 150 & 0.00682 & 1.561 & 0.518 & 3.171 \\ 200 & -0.111 & 2.080 & 0.515 & 3.898 \\ 250 & -0.194 & 2.603 & 0.513 & 4.585 \\ 300 & -0.258 & 3.136 & 0.512 & 5.222 \\ 350 & -0.311 & 3.678 & 0.512 & 5.793 \\ 400 & -0.356 & 4.224 & 0.512 & 6.272 \\ 450 & -0.395 & 4.775 & 0.511 & 6.641 \\ 500 & -0.429 & 5.328 & 0.511 & 6.903 \\ 600 & -0.488 & 6.440 & 0.511 & 7.289 \\ 700 & -0.548 & 7.556 & 0.511 & 7.643 \\ 800 & -0.583 & 8.674 & 0.510 & 7.983 \\ 900 & -0.620 & 9.797 & 0.510 & 8.281 \\ 1000 & -0.654 & 10.90 & 0.510 & 8.576 \\ & & & & \end{array}$


Table IV. Continued.

\begin{tabular}{ccccc}
\hline$R e$ & $x_{S} / H$ & $x_{r} / H$ & $y_{s} / H$ & $y_{r} / H$ \\
\hline (d) $\beta=0.7$ & & & & \\
50 & 0.238 & 0.788 & 0.538 & 1.490 \\
100 & -0.0710 & 1.428 & 0.528 & 2.442 \\
150 & -0.211 & 1.965 & 0.524 & 3.266 \\
200 & -0.304 & 2.490 & 0.521 & 4.028 \\
250 & -0.373 & 3.014 & 0.520 & 4.745 \\
300 & -0.429 & 3.539 & 0.519 & 5.414 \\
350 & -0.475 & 4.062 & 0.518 & 6.022 \\
400 & -0.516 & 4.581 & 0.518 & 6.543 \\
450 & -0.553 & 5.096 & 0.517 & 6.940 \\
500 & -0.584 & 5.607 & 0.517 & 7.177 \\
600 & -0.639 & 6.610 & 0.516 & 7.389 \\
700 & -0.686 & 7.588 & 0.516 & 7.601 \\
& & & & \\
(e) $\beta=0.9$ & & & & \\
50 & 0.0223 & 0.938 & 0.548 & 1.347 \\
100 & -0.207 & 1.244 & 0.532 & 2.312 \\
150 & -0.329 & 1.501 & 0.527 & 3.107 \\
200 & -0.412 & 1.734 & 0.525 & 3.805 \\
250 & -0.476 & 1.951 & 0.524 & 4.435 \\
300 & -0.529 & 2.158 & 0.523 & 4.996 \\
350 & -0.574 & 2.355 & 0.522 & 5.476 \\
400 & -0.612 & 2.542 & 0.521 & 5.852 \\
450 & -0.646 & 2.716 & 0.521 & 6.101 \\
500 & -0.677 & 2.879 & 0.520 & 6.348 \\
600 & -0.730 & 3.171 & 0.519 & 6.448 \\
700 & -0.775 & 3.427 & 0.519 & 6.657 \\
\hline
\end{tabular}

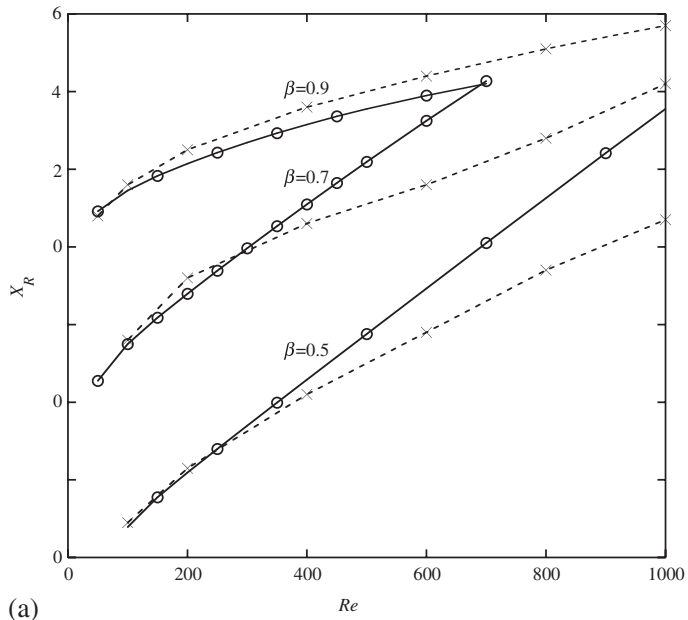

(a)

Figure 4. Variation of the normalized recirculation length with Reynolds number in the main duct (a) and the branch duct (b). Full line (our predictions) and dashed line [30]. Note: the vertical axis for each set of curves has a constant shift to avoid cluttering.

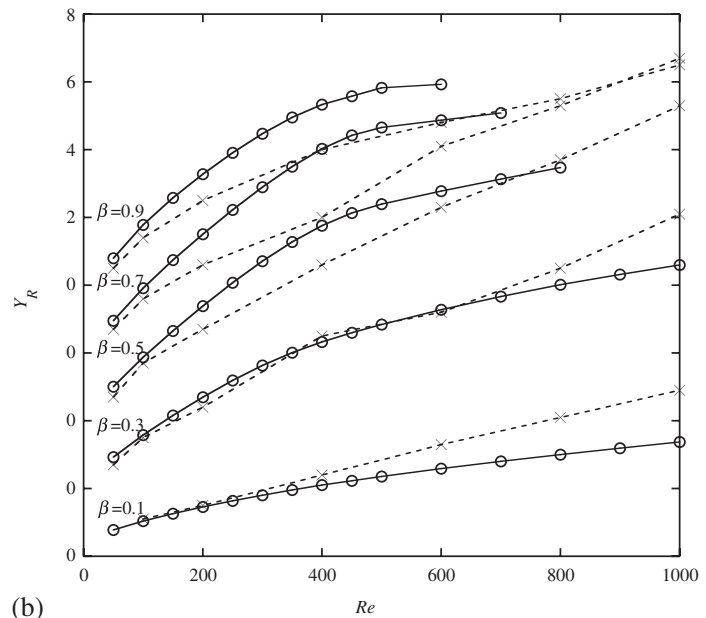

(b) 
Inspection of Figure 4 reveals that the length of the main recirculation increases both with the Reynolds number and the flow rate ratio. For the secondary recirculation its length also increases with Reynolds number, but has a non-monotonic behaviour with $\beta$. At low values of $\beta$ the recirculation increases, followed by a decrease at higher flow rate ratios. Note that an increase in the value of $\beta$ effectively represents a decrease in the flow rate exiting the main duct.

In the branch duct, the flow separates immediately after the upstream inlet corner and the increase in recirculation length with Reynolds number is essentially due to the corresponding downstream motion of the reattachment point, as can be verified in Table IV. On the other hand, for the secondary eddy attached to the lower horizontal wall, not only the reattachment point moves further downstream with Reynolds number but also the separation point actually moves upstream, both contributing to an increase in recirculation length. However, the effect of flow rate ratio upon $x_{s}$ and $x_{r}$ is not always the same: at a constant Reynolds number, the separation point always moves upstream with increasing values of $\beta$, whereas the reattachment location moves downstream for values of $\beta$ up to 0.5 and then move backwards, in the upstream direction, for values of $\beta>0.5$. The consequence is an increase in $X_{R}$ for $\beta<0.5$, followed by a decrease in $X_{R}$ for $\beta>0.5$.

\subsection{Assessment of the time discretization method: pulsating laminar channel flow of Newtonian fluids}

To assess the performance of the time discretization scheme and in particular to quantify the uncertainty of the time-dependent calculations as a function of the time step, predictions were made for pulsating laminar channel flow of Newtonian fluids generated by an imposed, sinusoidal pressure gradient of the form:

$$
-\frac{1}{\rho} \frac{\mathrm{d} p}{\mathrm{~d} x}=K_{s}+K_{\mathrm{O}} \cos (\omega t)
$$

where $-\rho K_{\mathrm{O}}$ is the amplitude of the oscillating pressure gradient of frequency $\omega$ superimposed in a steady pressure gradient of magnitude $-\rho K_{s}$. A non-dimensional frequency is usually expressed as the Womersley number, $\alpha=h / \sqrt{v / \omega}$ where $v=\eta_{0} / \rho$, and the period of the oscillation $T_{\mathrm{O}}=2 \pi / \omega$ will serve as time scale in the next figures. This case has an analytical solution, which can be found in the literature $[49,50]$. In the Appendix at the end, we will give the main results of that solution, which are useful for the comparison with predictions of the present section. The flow geometry here corresponds to the inlet plane to the T-junction (see Figure 1), a planar channel with half-height $h=H / 2$. The origin of the coordinate system is at the symmetry plane and the transverse coordinate is $y$.

Numerical calculations of this pulsating channel flow were carried out for the same flow conditions of Khodadadi et al. [31] and Khodadadi [32] in their T-junction in order to prepare for the next set of simulations of the pulsating laminar flow in a $90^{\circ}$ T-junction of Section 3.3. The Reynolds number based on the steady bulk flow in the channel $\left(\bar{u}_{s} \equiv \bar{u}_{1}\right.$, see Appendix $)$ and the wall-to-wall distance $(2 h)$ was 102 , the Womersley number $\alpha=4.864$, the frequency $f$ of the oscillating pressure gradient was set to $f=1.1$ cycles/s $\left(\omega=2 \pi f=2.2 \pi \mathrm{s}^{-1}\right)$ and its amplitude was $\rho K_{\mathrm{O}}=190 \mathrm{~Pa} / \mathrm{m}$, with the steady pressure gradient set at $\rho K_{s}=75.1 \mathrm{~Pa} / \mathrm{m}$. The fluid density was $\rho=1150 \mathrm{~kg} / \mathrm{m}^{3}$ and its kinematic viscosity was $v=0.73 \times 10^{-5} \mathrm{~m}^{2} / \mathrm{s}$.

In Figures 5 and 6 predictions of the normalized velocity and shear stress profiles are compared with the corresponding theoretical results, respectively. The crosses (X) and the thick lines pertain to steady flow, and the thin lines and other symbols to the unsteady flow. The mesh used had 100 


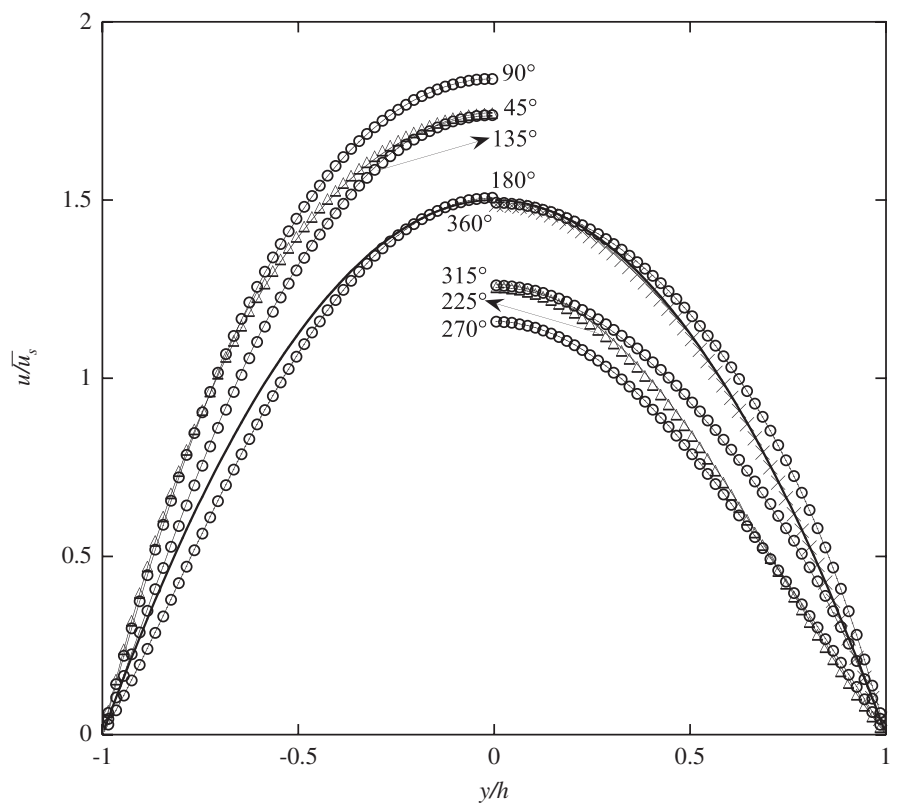

Figure 5. Comparison between analytical (lines) and computed (symbols) velocity profiles for Newtonian channel flow: steady flow (thick line and X); unsteady flow within a cycle, $\alpha=4.864, K_{\mathrm{O}} / K_{s}=2.530$ (thin line; $\Delta$ represent data at 45 and $225^{\circ}$ ). Only half the data were plotted. Note: To avoid cluttering half, the profiles were plotted at $y / h>0$, the other half at $y / h<0$.

control volumes in the transverse direction. Following standard practice in channel flow analysis, the velocity plotted was normalized by the bulk flow velocity $\left(u / \bar{u}_{1}\right)$ and the shear stress $\left(\tau_{x y}\right)$ with $\frac{1}{3}$ of the wall shear stress $\left(\tau_{x y} /\left[\eta \bar{u}_{1} / h\right]\right)$. The theoretical stress is given by Equation (A5) in the Appendix.

For steady flow the agreement is excellent, with errors not exceeding $0.9 \%$ and shows that the mesh is adequate in terms of spacing.

Unsteady flow calculations were carried out with the same mesh and a time step normalized with the oscillating period $\Delta t / T_{\mathrm{O}}=0.01$, where $T_{\mathrm{O}}=2 \pi / \omega$. Comparison between the theoretical and predicted velocity profiles along a complete cycle is excellent, as shown also in Figure 5, suggesting that a normalized time step of $1 \%$ of the period of an oscillation is adequate for accurate unsteady flow predictions. It is emphasized that this conclusion only holds because the temporal scheme is second-order accurate. The profiles at opposite parts of the cycle are not identical as is clearly seen in the comparison between the profiles for steady flow and for 180 and $360^{\circ}$. When the flow is decelerating $\left(180^{\circ}\right)$ the profile is sharper, whereas it tends to be more full when the flow accelerates $\left(360^{\circ}\right)$. The comparison between the predicted and analytical normalized shear stress profiles is shown in Figure 6 and the match between both sets is again excellent.

The pressure gradient has a minimum at $\omega t=180^{\circ}$, a maximum at $360^{\circ}$, and is zero at $\omega t=0$ and $90^{\circ}$. Inspection of the velocity profiles in Figure 5 shows that the velocity is out of phase with the pressure gradient by about $90^{\circ}$. The evolution with time of the calculated and theoretical maximum $\left(u_{c} / \bar{u}_{s}\right)$ and bulk $\left(\bar{u} / \bar{u}_{s}\right)$ velocities is plotted in Figure 7 in normalized form and the comparison is 


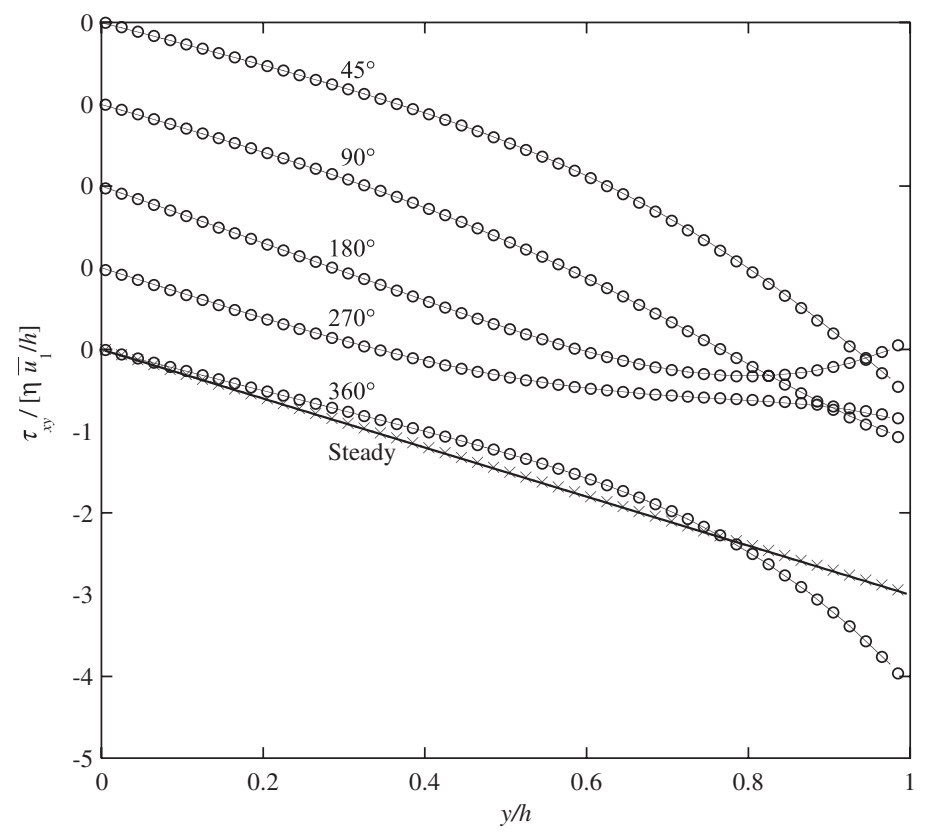

Figure 6. Comparison between analytical (lines) and computed (symbols) shear stress profiles for Newtonian channel flow: steady flow (thick line and X); unsteady $\alpha=4.864, K_{\mathrm{O}} / K_{s}=2.530$ (thin line and $\mathrm{O}$ ). Only half the data were plotted. Note: the vertical axis for each curve has a constant shift to avoid cluttering.

again excellent. The phase angle between the bulk velocity and the pressure gradient $\left(\delta^{*}\right)$ is given by Equation (A8) and for the present flow conditions $\delta^{*}=80.4^{\circ}$. The predicted phase angle $\delta^{*}$ has a small error, which is perfectly within the time step size, $\Delta t / T_{\mathrm{O}}=0.01 \Rightarrow$ angle step of $3.6^{\circ}$.

\subsection{Unsteady diverging laminar flow of Newtonian fluids in a $90^{\circ}$ T-junction}

Flow conditions here are the same as in Khodadadi et al. [31] and Khodadadi [32] and we compare our predictions with the experimental data of the former authors, who conducted experiments using a mixture of water and glycerine. The fully developed periodic Newtonian velocity profile given by Equations (A3)-(A5) was imposed at the inlet, but we note that in the calculations of Khodadadi et al. [31] their pulsatile inlet profile corresponded to the measured inlet profile at $x / h=-3.5$, which is not always identical to the sum of Equations (A3) and (A5). Otherwise flow parameters are the same as in Section 3.2, i.e. $R e=102, \alpha=4.864$ and $K_{\mathrm{O}} / K_{s}=2.530$, complemented by the flow rate ratio which here was set to $\beta=0.7$ following the experimental case of Khodadadi.

Mesh M2, with 40 uniformly distributed cells (40U) in the transverse direction $(\Delta y / H=0.025)$, and mesh M4, with the same number of 40 cells, but non-uniformly distributed for finer spacing close to the walls $\left(\Delta y_{\min } / H=0.01\right)$, were used. The results to be presented were normalized as follows: distances with $H$ (cf. Figure 1), velocities by the steady flow bulk velocity $\bar{u}_{s}$, time by the imposed pulsating period $2 \pi / \omega$ and stresses by the wall shear stress under steady flow conditions, $6 \eta \bar{u}_{s} / H$. 


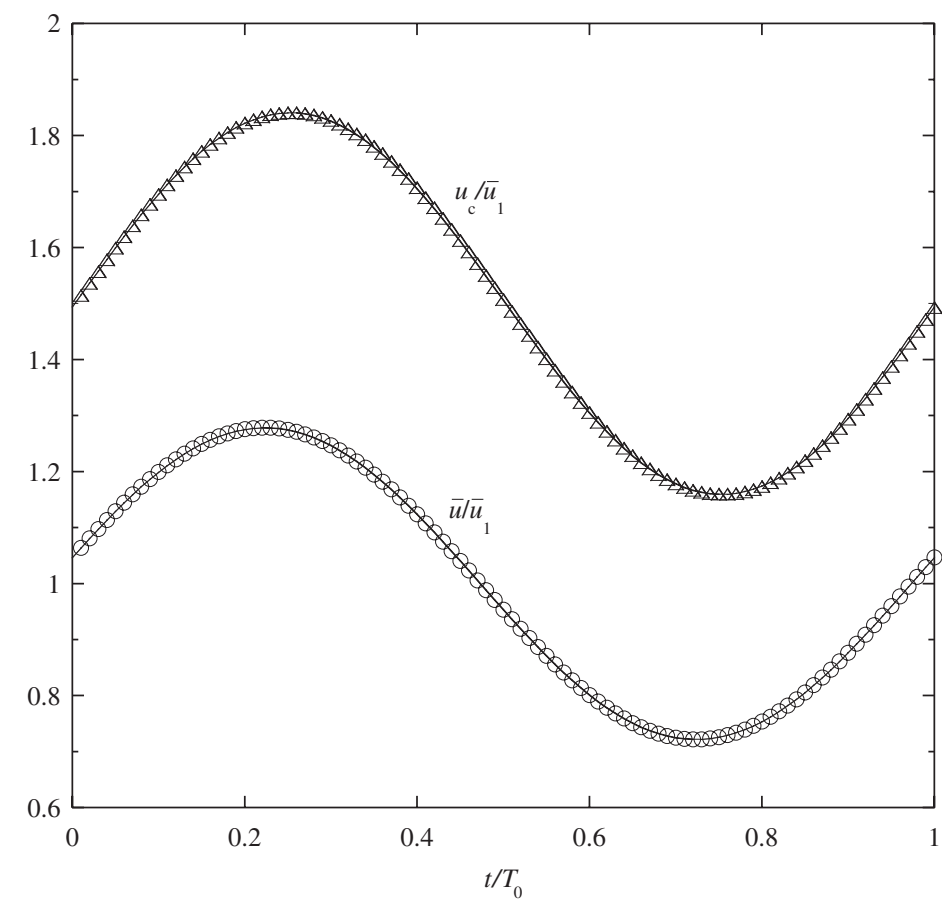

Figure 7. Evolution with time of the centre-plane $\left(\Delta, u_{c} / \bar{u}_{1}\right)$ and space-averaged bulk $\left(\mathrm{O}, \bar{u} / \bar{u}_{1}\right)$ velocities, $\alpha=4.864, K_{\mathrm{O}} / K_{s}=2.530$. Calculations with $\Delta t / T_{\mathrm{O}}=0.01$, symbols; theory, lines.

As sketched in Figure 1 and in accordance with the steady flow results of Section 3.1, the unsteady flow in the T-junction also has a primary recirculation attached to the upstream wall of the branch duct and a secondary recirculation at the lower wall of the main duct. The precise location of these regions and their time evolution are fundamental to understand the relationship between hemodynamics and vascular diseases. Figure 8 indicates the progression with time of the coordinates of the separation and reattachment points of both recirculating regions during a complete cycle. Note that $X_{r}=x_{r} / H \neq X_{R}$.

Refinement of the mesh close to the bifurcation and walls does not significantly improve the prediction of the separation and reattachment points. Similarly, refinement of the time step from $\Delta t / T_{\mathrm{O}}=0.01$ to $\delta t^{*}=0.001$ only improves the prediction of the sudden variations of the plotted quantities because of the better resolution and it is remarkable that the sudden reduction in the main eddy size is equally well predicted when using a coarser time step which is 10 times larger than the finer time step, thus showing the advantages of the second-order time discretization method. Unless otherwise stated, henceforth the results to be presented rely on mesh M4 and a time step of 0.01 , which is perfectly adequate to obtain accurate values. This more refined mesh near the wall was selected because we wanted to use the same mesh here and in the non-Newtonian predictions of Section 3.4, where the fluid is expected to exhibit larger velocity gradients near the walls on account of shear thinning.

The secondary separation (separation in the main duct) starts after the pressure gradient goes through a maximum and grows in length when the pressure gradient is already decreasing. The 


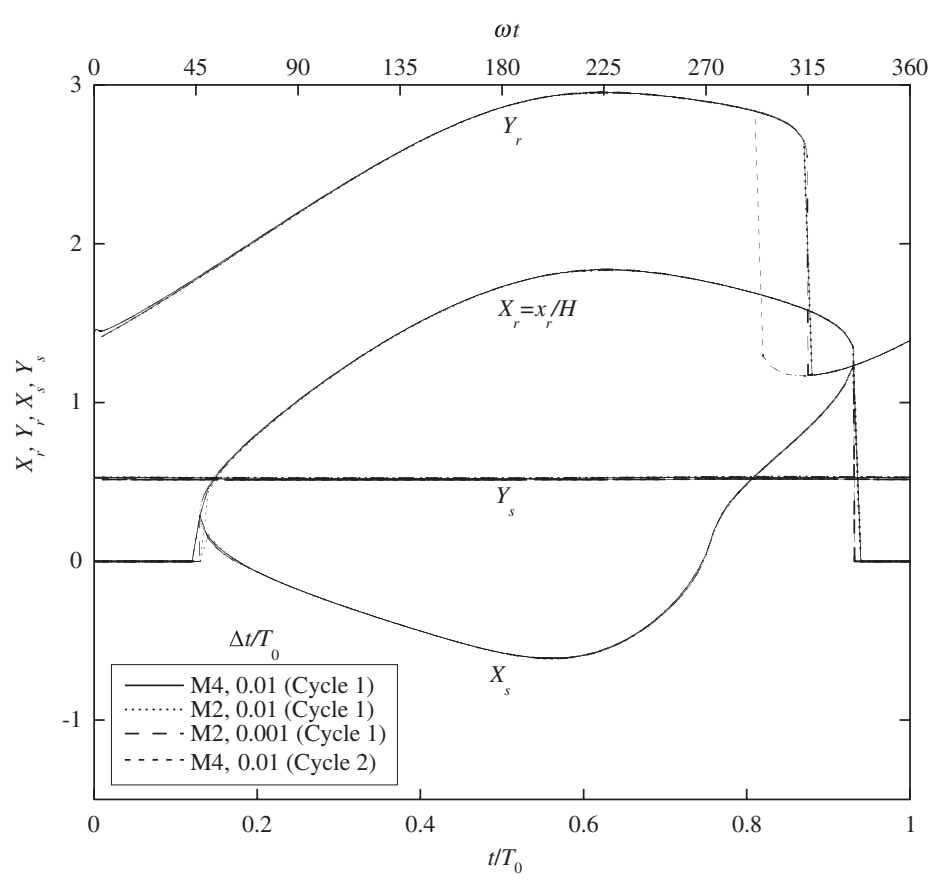

Figure 8. Evolution with time of the loci of beginning and end of the two recirculations for Newtonian flow. Effects of mesh refinement using uniform (M2) and non-uniform (M4) meshes, of time-step size $\left(\Delta t / T_{\mathrm{O}}\right)$ and cycles in time $\left(R e=102, \alpha=4.864, K_{\mathrm{O}} / K_{s}=2.530\right)$.

effect of inertia is clear in the initial acceleration of the flow, when it goes through an adverse pressure gradient and comes back to a phase of favourable pressure gradient. The growth of the secondary eddy is both due to the upstream movement of the separation point and the downstream motion of the reattachment point, as commented above for the steady flow condition. For a while (scaled time ranging from around 0.93 to 0.13 in the next cycle), there is no secondary recirculating region. In contrast, the main separation, which starts at the corner of the branch duct, is always present. It grows with a delay relative to the pressure gradient until the moment when it suddenly shrinks to a small size to reinitiate the cycle. This abrupt length reduction is due to a physical and mathematical instability associated with the non-linearity of the phenomenon, because the precise moment when the recirculation shortens varies as more flow periods are simulated suggesting that this problem has two solutions, as also shown in the comparison between the curves for cycle 1 and cycle 2 in Figure 8. What we denote here by 'cycle 1' and 'cycle 2' refer to two fully established situations obtained after running the simulations for several periods (more than 30 ) of the imposed pulsating pressure gradient. 'Cycle 1' occurs first and the numerical solution appears to be invariant with time for a number of cycles (2-4). However, as discussed below, the solution bifurcates to the situation denoted 'cycle 2', which therefore seems more stable than 'cycle 1' and remains time invariant after that. This bifurcation was found to exist with both meshes (M2 and M4) as well as for different time steps, so it is not a numerical artefact.

The normalized critical time at which an abrupt reduction is recorded in the main recirculation size is initially equal to 0.86 (cycle 1), but at later times it suddenly drops to 0.81 (cycle 2) and remains at that value henceforth. Both this unstable behaviour and the sudden decrease in $Y_{r}$ have 


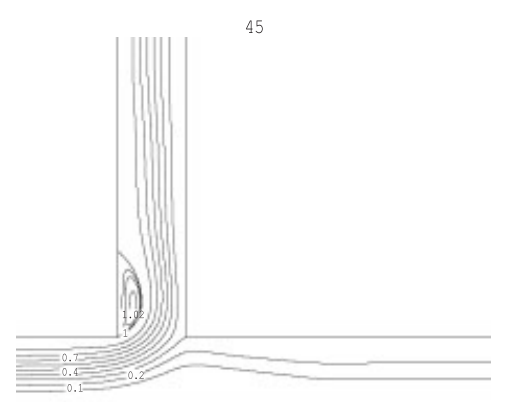

90

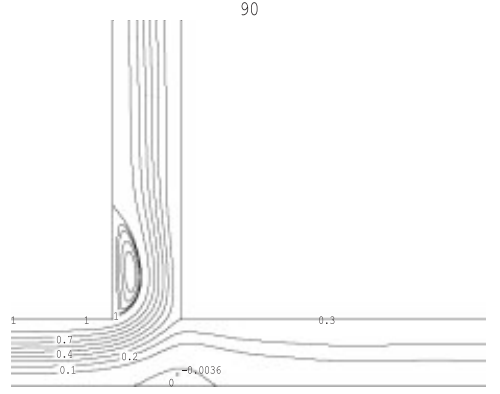

135

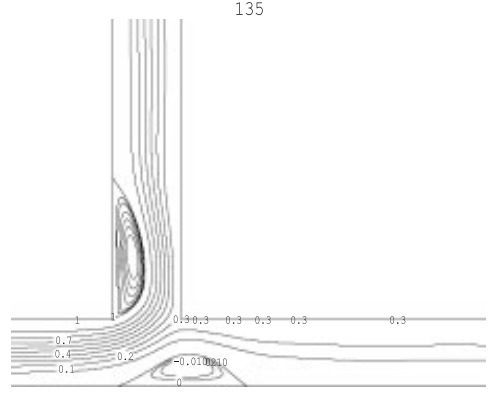

180

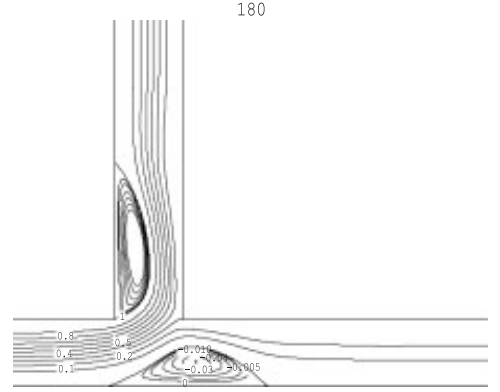

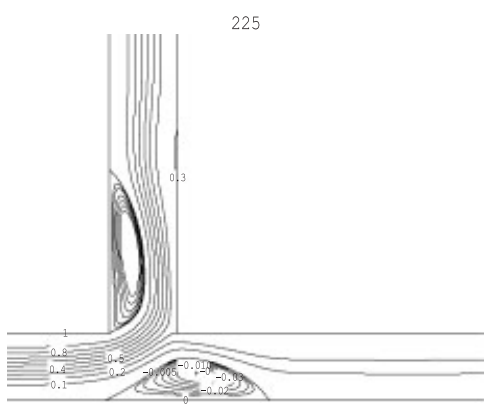

270

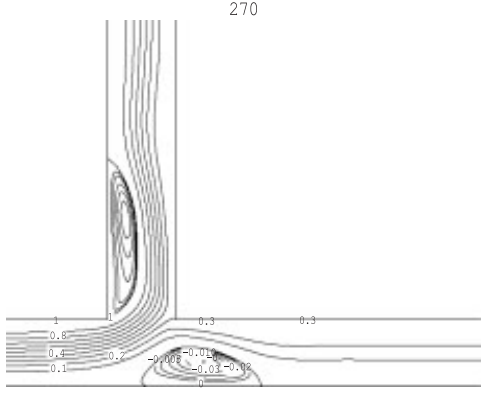

315

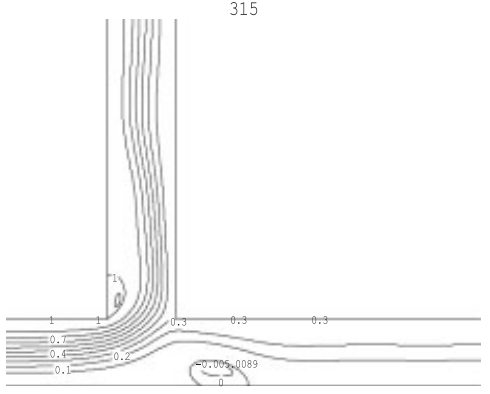

360

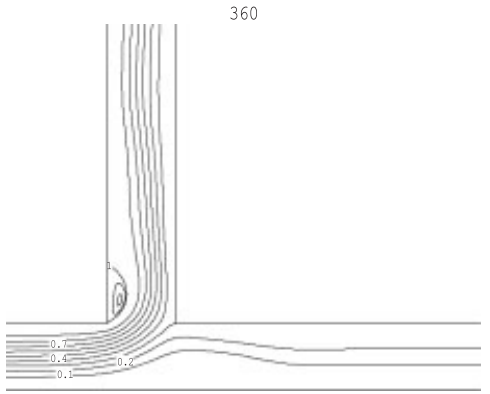

Figure 9. Sequence of instantaneous streamlines within a cycle (numbers on top refer to $\omega t$ ) obtained on mesh M4 and $\Delta t / T_{\mathrm{O}}=0.01\left(R e=102, \alpha=4.864, K_{\mathrm{O}} / K_{s}=2.530\right)$. 

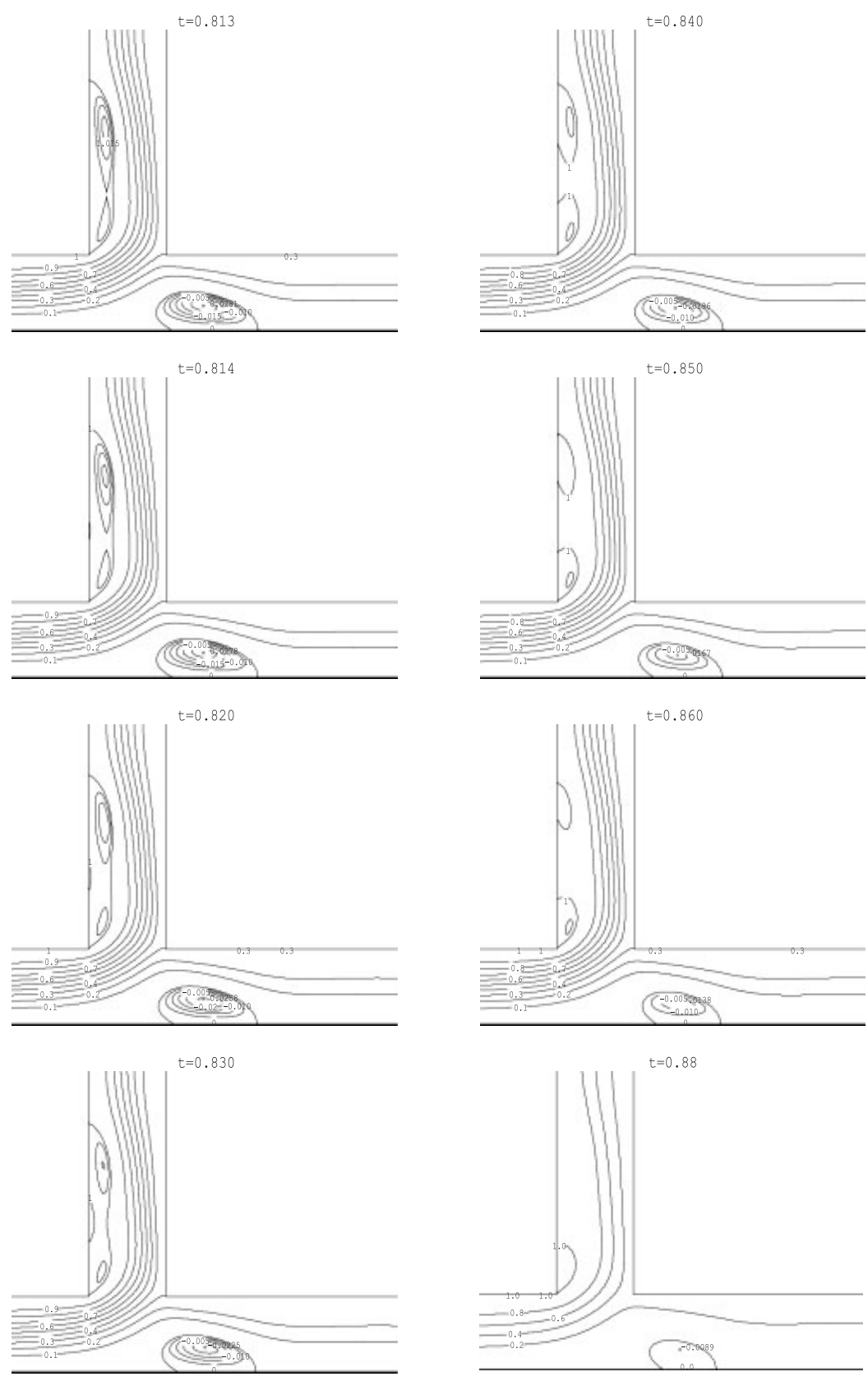

Figure 10. Zoom of instantaneous streamlines in the range $t / T_{\mathrm{O}} \in[0.813,0.88]$. Calculations using $\Delta t / T_{\mathrm{O}}=0.001\left(R e=102, \alpha=4.864, K_{\mathrm{O}} / K_{s}=2.530\right)$. 


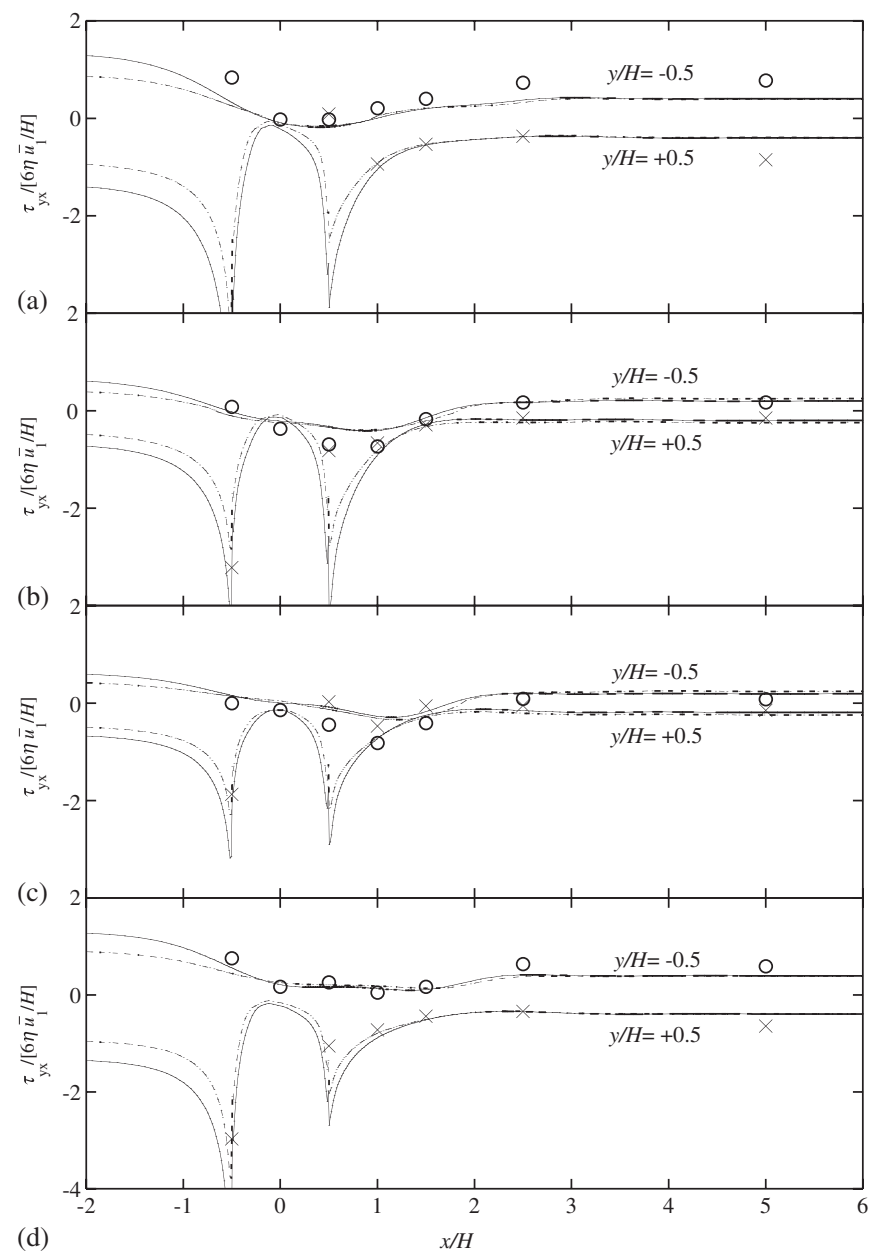

Figure 11. Variation within a cycle of the shear stress along the lower $(y / H=-0.5)$ and upper $(y / H=0.5)$ walls. Symbols pertain to Newtonian experimental data from Khodadadi [32]; solid lines, Newtonian predictions; dashed lines, non-Newtonian predictions: (a) $\omega t=90^{\circ}$; (b) $\omega t=180^{\circ}$; (c) $\omega t=270^{\circ}$; and (d) $\omega t=360^{\circ}$.

not been previously reported, as can be confirmed by inspection of Figure 16(b) of Khodadadi et al. [31]. The evolution of the two vortices within a cycle is depicted by the streamline plots in Figure 9 . Notice the absence of the secondary eddy between 0 and $45^{\circ}$ and the small main eddy at $315^{\circ}\left(t / T_{\mathrm{O}}=0.875\right)$, which is the result of events starting shortly after $270^{\circ}\left(t / T_{\mathrm{O}}=0.75\right)$.

To investigate in more detail the sudden reduction in size of the main eddy, the streamlines have been zoomed in space and time and are shown in Figure 10. The plots correspond to the behaviour denoted 'cycle 2', which refers to the more stable solution, and were obtained with mesh M4, but using a higher temporal resolution of $\Delta t / T_{\mathrm{O}}=0.001$. According to Figure 8 , the sudden drop in eddy size occurs at instant $t_{\mathrm{cr}}=0.81 T_{\mathrm{O}}$ based on a time step of 0.01 , but it is more correctly calculated to be 0.814 using the time step of 0.001 . Careful inspection of Figure 10 shows that the 


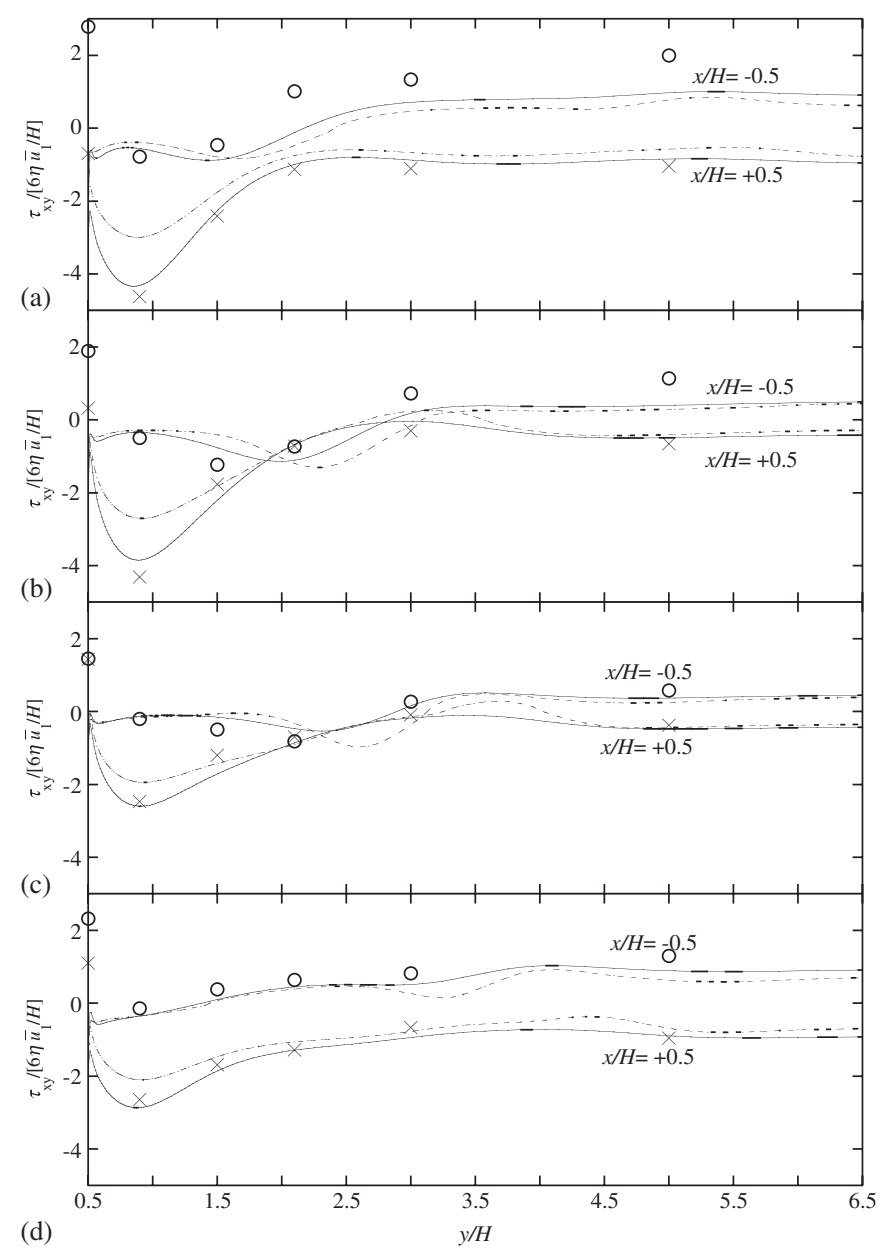

Figure 12. Variation within a cycle of the shear stress along the upstream $(x / H=-0.5)$ and downstream $(x / H=0.5)$ walls. Symbols pertain to Newtonian experimental data from Khodadadi [32]; solid lines, Newtonian predictions; dashed lines, non-Newtonian predictions: (a) $\omega t=90^{\circ}$; (b) $\omega t=180^{\circ}$; (c) $\omega t=270^{\circ}$; and (d) $\omega t=360^{\circ}$.

main vortex separates from the wall and breaks into two vortices, with the downstream part being shed, leading to the unstable and discontinuous behaviour of this vortex. Therefore, the behaviour of the main and secondary eddies is quite distinct and this situation is expected to occur in strong bifurcations. Henceforth, and unless otherwise stated, the remaining figures pertain to the more stable flow condition, 'cycle 2'.

Another result of relevance to hemodynamics is the evolution of the shear stress with time. The predicted profiles of $\tau_{x y}$ scaled with inlet wall values $\left(\tau_{x y} /\left(6 \eta \bar{u}_{s} / H\right)\right)$ are plotted as solid lines in Figures 11 and 12 and compared with experimental data (symbols) presented by Khodadadi [32]. Figure 11 plots data along the lower and upper walls of the main duct $(y / H=-0.5$ and +0.5 , respectively) and Figure 12 does the same along the upstream and downstream walls of the 

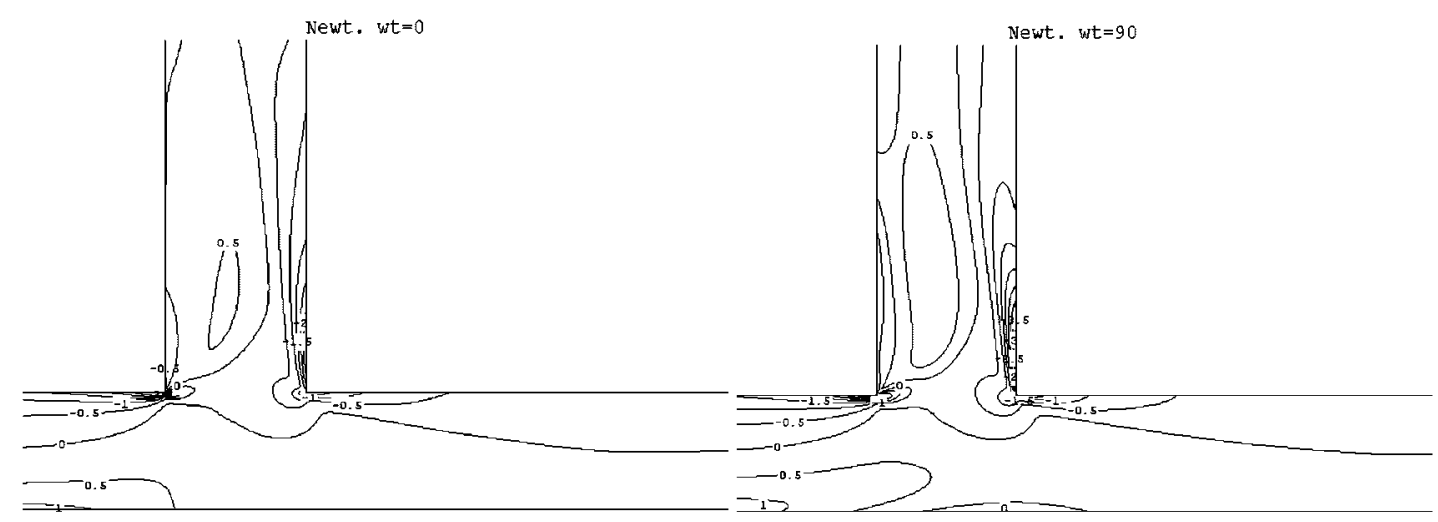

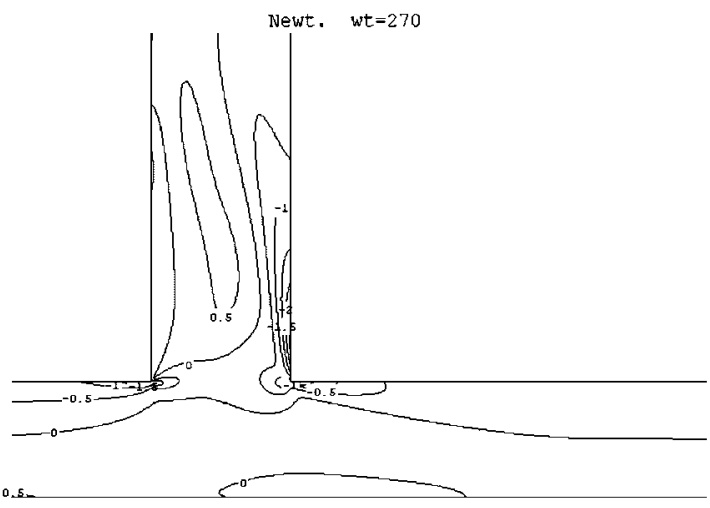

Figure 13. Sequence of instantaneous contour maps of normalized shear stress $\left(\tau_{x y} /\left(6 \eta \bar{u}_{1} / H\right)\right)$ within a cycle (numbers on top refer to $\omega t$ ) obtained on mesh $\mathrm{M} 4$ and $\Delta t / T_{\mathrm{O}}=0.01$ for Newtonian flow.

branch duct $(x / H=-0.5$ and +0.5 , respectively). The dashed lines pertain to predictions for non-Newtonian fluids to be discussed in the next section.

Our calculations of the shear stress for Newtonian fluids are in reasonable agreement with the experimental data and a couple of issues should be discussed before proceeding. First, the imposed inlet condition for our calculations correspond to a pulsatile fully developed flow and this does not match closely the measured inlet condition, as already mentioned. Secondly, Khodadadi [32] did not measure directly the shear stresses on the walls, but calculated them from the measurements of the tangential velocity component close to the wall; therefore, his stress data do not include contributions from the normal gradient of the transverse velocity component, which is not negligible near the branch and where there is flow separation and reattachment. The two sharp negative peaks in the plots of Figure 11 mark the corners of the branch pipe and between them there is no wall.

The biggest discrepancy between the predicted and measured shear stresses is seen where the pressure gradient changes from favourable to adverse, a feature that does not come as a surprise, given the limitations of the measurements alluded above. Generally, the evolution of the shear stress along the walls is well correlated with flow separation, precisely the recirculating zones where the lowest stresses are seen. These are the regions of possible formation and accumulation of lipids and blood clots leading to atherosclerosis [51]. On the walls opposing the vortices, maximum stresses 
are observed, especially where the flow impinges the wall, and these higher stresses are responsible for the deterioration of the endothelium of arteries. High stresses can also rupture red blood cells releasing haemoglobin in the blood $[52,53]$. It is therefore pretty clear that an adequate assessment of shear stress effects on blood vessel walls relies heavily on the exact prediction of recirculation flow sizes. Thus, the benchmark data of Section 3.1.2 are amply justified: any predictive code will need to be verified against that data before embarking in more complex three-dimensional simulations.

Within the flow domain the evolution of the normalised $x y$ shear stress with time is depicted in the instantaneous contour plots of the calculated shear stress of Figure 13 showing that the maximum stresses indeed take place at walls and the low stresses within the separated flow regions. It can also be observed how the dynamics of the main recirculation in the branch along one period (cf. Figure 9) influences the shear stress distribution in that area of the flow and on the upstream wall. Owing to the symmetry of the stress tensor $\tau_{x y}=\tau_{y x}$, the interpretation of the shear forces acting on the duct walls is straightforward after the consideration of the commonly accepted stress convention.

A final more detailed comparison between our numerical predictions and the comprehensive experimental data of Khodadadi et al. [31] is presented in the plots of Figures 14 and 15, showing transverse profiles of the streamwise velocity in the main and branch ducts, respectively. These data were again obtained on mesh M4 using $\Delta t / T_{\mathrm{O}}=0.001$, whereas the experimental data are the raw data provided by Prof. Khodadadi, here used without any post-processing.

Predictions are remarkably close to the experimental data at all times within the cycle, with some small differences traced back to the different inlet conditions, and the comparison is actually better than between the experiments and the calculations performed in their original paper [31].

\subsection{Unsteady diverging laminar flow of a generalized Newtonian fluid in a $90^{\circ}$ T-junction}

To evaluate the effect of a non-linear viscosity on the flow characteristics, numerical predictions of the unsteady flow in the $90^{\circ} \mathrm{T}$ bifurcation were carried out for a Carreau fluid model under the same conditions as in Section $3.3(\beta=0.7)$. The fluid properties selected were those of Banerjee et al. [54], who fitted the CY model (Equation (5)) to the experimental blood data of Cho and Kensey [55] to obtain: $n=0.3568$; Carreau parameter $a=2$; zero shear rate viscosity, $\eta_{0}=0.056 \mathrm{Pas}$; infinite shear rate viscosity, $\eta_{\infty}=0.00345$ Pas; and time constant $\lambda=3.313 \mathrm{~s}$. In these calculations the viscosity of the fluid varies from point to point and is calculated locally with Equation (5). From these values, and for the same flow rate and $\beta$ of previous section, it is possible to estimate average shear rates at the inlet and outlet branch ducts and the corresponding average viscosities given by

$$
\begin{aligned}
& \overline{\dot{\gamma}}_{1}=\frac{\bar{u}_{1}}{0.5 H}=14.9 \mathrm{~s}^{-1} \rightarrow \bar{\eta}_{1}=0.0073 \mathrm{Pas} \\
& \overline{\dot{\gamma}}_{3}=\frac{\bar{u}_{3}}{0.5 H}=10.4 \mathrm{~s}^{-1} \rightarrow \bar{\eta}_{3}=0.0088 \mathrm{Pas}
\end{aligned}
$$

These viscosity values are close to the constant viscosity used in the Newtonian calculations of Sections 3.2 and $3.3(\eta=0.0084 \mathrm{Pas})$; hence, their results are comparable with the CY results of this section.

On the basis of numerical tests, the influence of both mesh and time-step refinements on the quality of predictions for the Carreau fluid was found to be similar to those obtained with the 

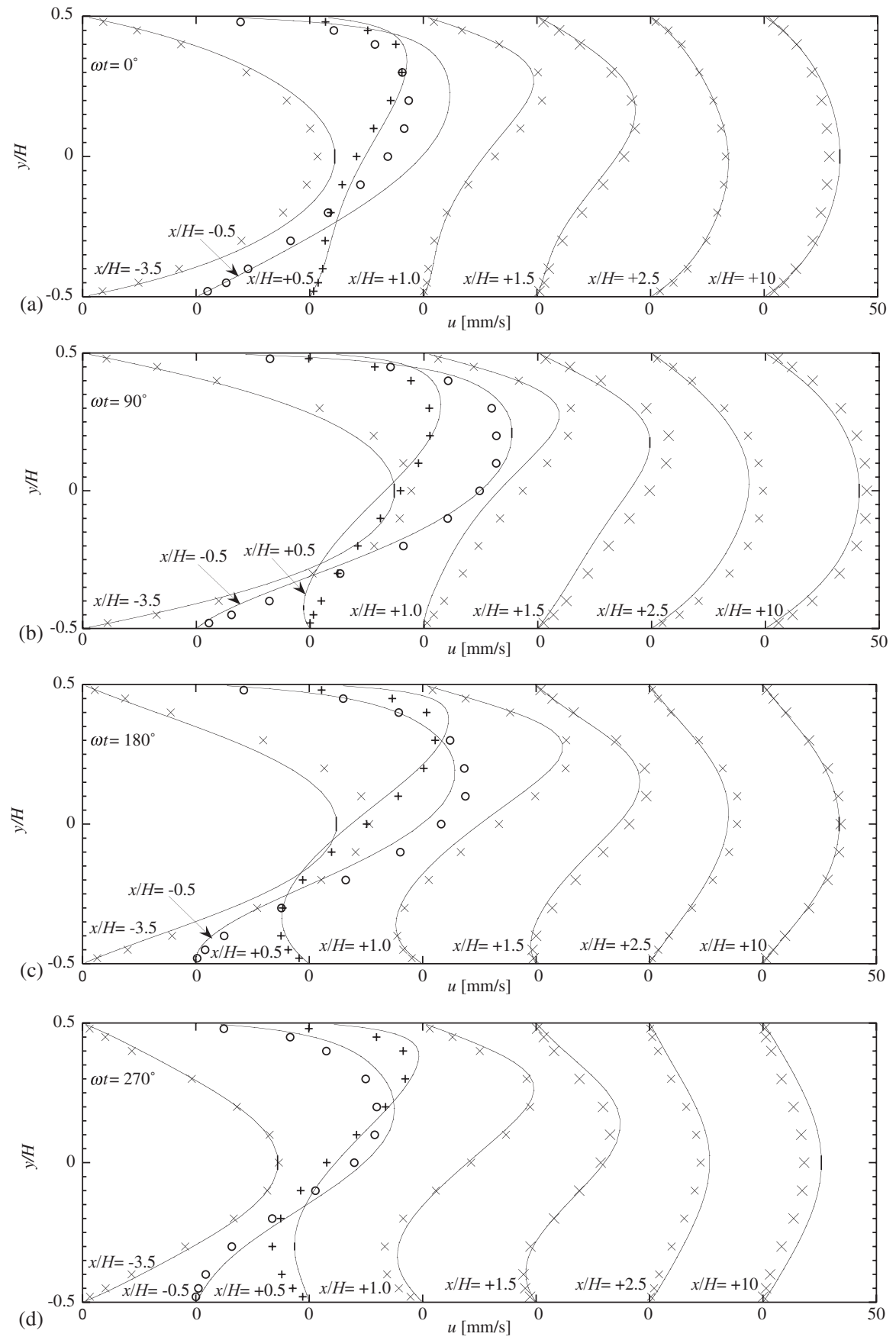

Figure 14. Transverse profiles of velocity in the main duct; predictions (lines), experimental data of Khodadadi (symbols) $\left(R e=102, \alpha=4.864, K_{\mathrm{O}} / K_{s}=2.530\right)$. 

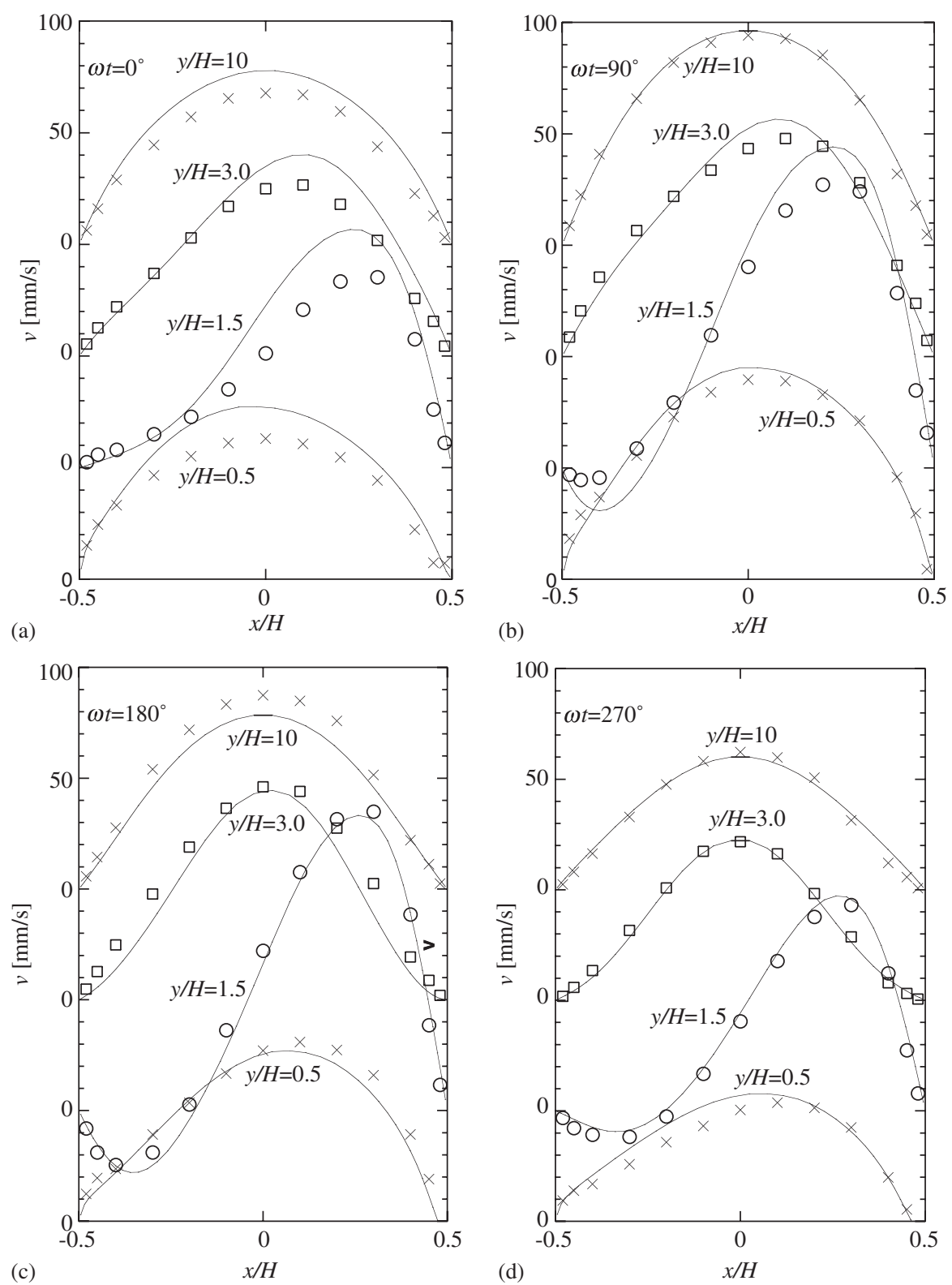

Figure 15. Transverse profiles of velocity in the branch duct: predictions (lines), experimental data of Khodadadi (symbols) $\left(R e=102, \alpha=4.864, K_{\mathrm{O}} / K_{s}=2.530\right)$.

Newtonian fluid; hence, mesh M4 was used here as well together with $\Delta t / T_{\mathrm{O}}=0.01$. The imposed inlet velocity profile for the Carreau fluid was the same fully developed profile used for the Newtonian flow simulations, at the same Womersley number (that is, Equations (A2) to (A4)). We have decided for this choice because there is no corresponding analytical solution for the CY 


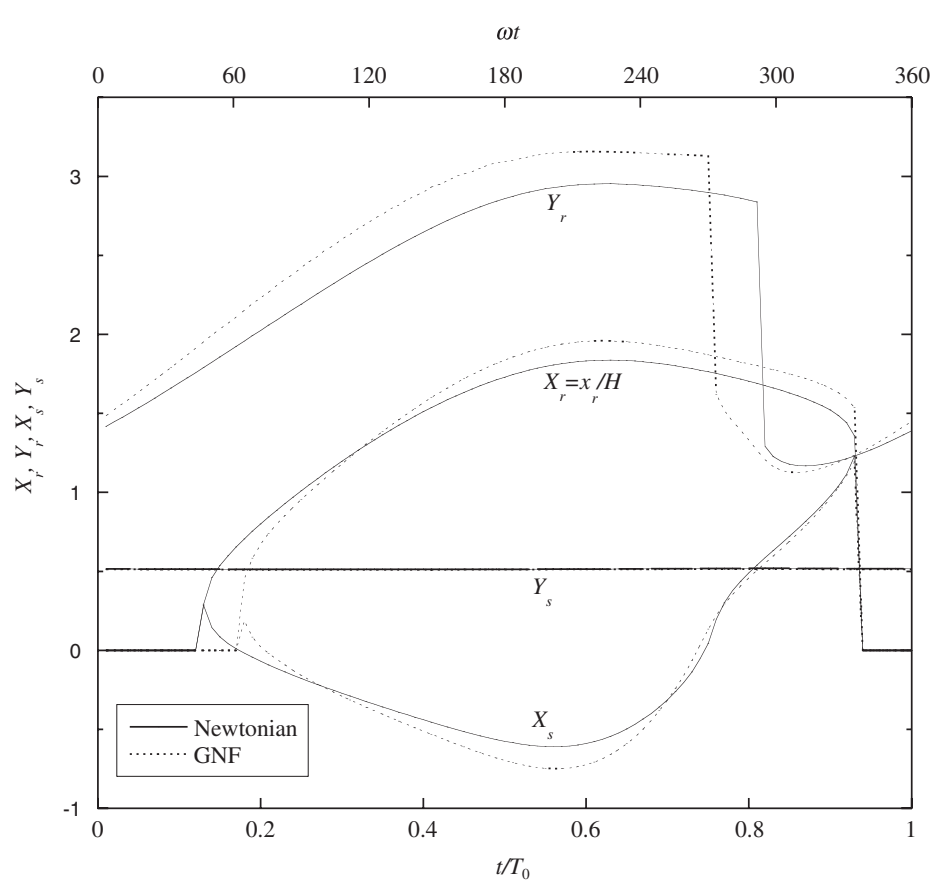

Figure 16. Beginning, end and size of the two recirculation regions in time cycle 2 for Newtonian and generalized Newtonian (Carreau-Yasuda model) fluids. Computations using the three-time level method and $\Delta t / T_{\mathrm{O}}=0.01$.

viscosity model and in this way one can compare the two cases (Newtonian and non-Newtonian) for exactly the same imposed inlet conditions.

Overall, the flow characteristics for the Carreau fluid were the same as for the Newtonian case, but longer recirculations were predicted on account of the lower viscosities found in high shear regions. Figure 16 plots the evolution within a period of the loci of the beginning and end of the two recirculation regions for a well-established situation after many initial periods; thus, under the conditions designated as 'cycle 2' in Section 3.3. Although the eddies are longer for the shear-thinning fluid, they also exist for a shorter period of time than the corresponding Newtonian eddies. As observed for the Newtonian fluid, the abrupt critical decrease in size of the main vortex is also seen here, but now it takes place earlier within the period, at around $270^{\circ}$. The secondary recirculation in the main duct also starts at a later time in the cycle, at $t / T_{\mathrm{O}} \approx 0.17$ against the Newtonian value of $t / T_{\mathrm{O}} \approx 0.12$. However, it is interesting to notice that this vortex, attached to the lower wall along the main duct, disappears at exactly the same moment along the period (at $t / T_{\mathrm{O}} \approx 0.93$ ) for the Newtonian and the non-Newtonian fluids. This may be explained by noticing that the reattachment point of this vortex is in a zone of very low-velocity gradients (see streamlines in Figure 9 for the Newtonian case); hence, the shear-rate dependency of viscosity is at work only marginally.

Generally speaking, the longer recirculations give rise to smaller normalized shear stresses at the walls, as can be seen in Figures 11 and 12, where the dashed lines pertain to the non-Newtonian fluid. For this fluid the stresses were normalized as for the Newtonian fluid, i.e. using $6 \eta \bar{u}_{1} / H$ 


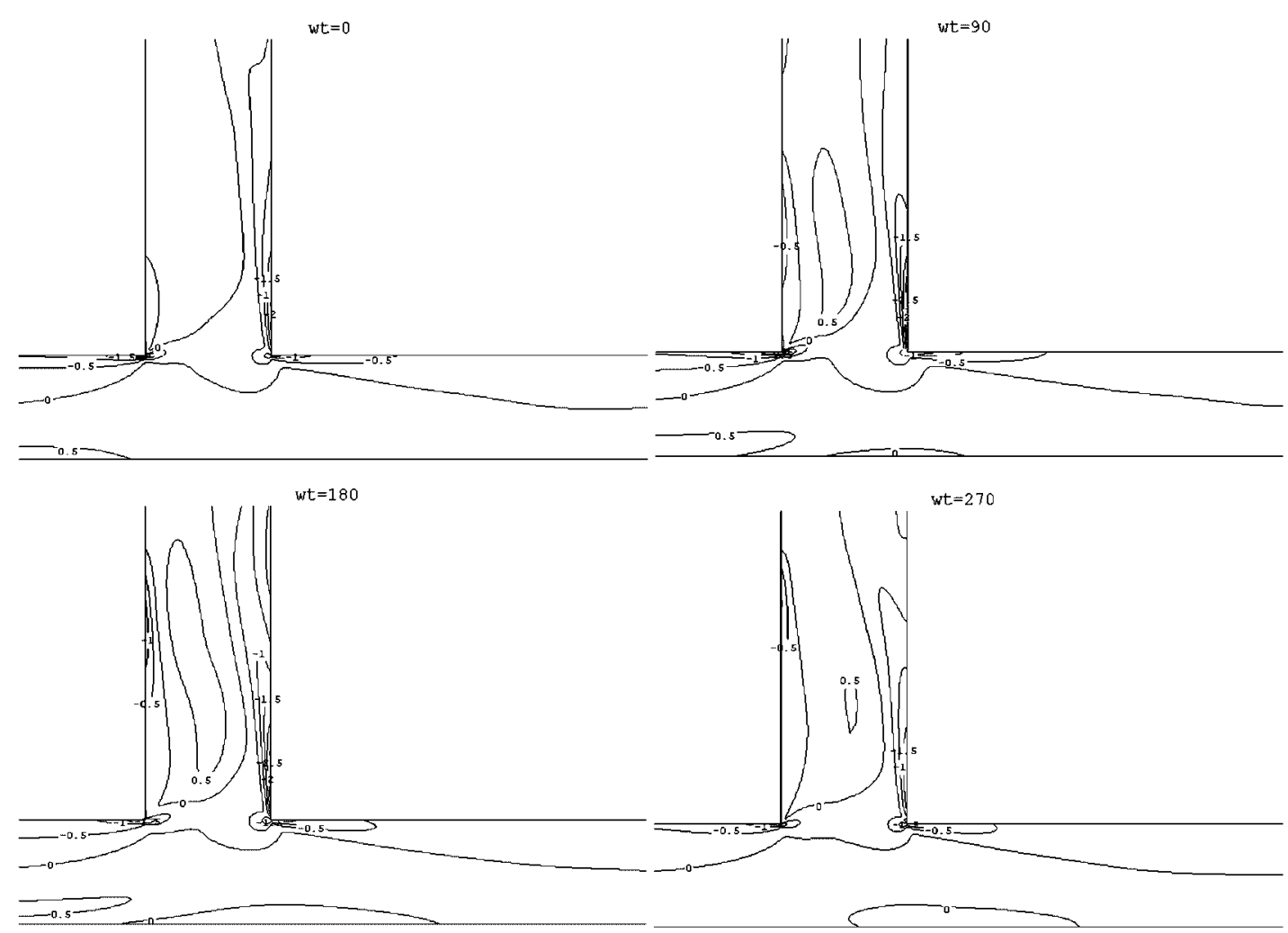

Figure 17. Sequence of instantaneous contour maps of normalized shear stress $\left(\tau_{x y} /\left(6 \eta \bar{u}_{1} / H\right)\right)$ within a cycle (numbers on top refer to $\omega t$ ) obtained on mesh M4 and $\Delta t / T_{\mathrm{O}}=0.01$ for non-Newtonian flow.

where $\eta=0.0084 \mathrm{Pas}$, the Newtonian viscosity value. Although this is rather arbitrary, this viscosity value represents well a viscosity at an average shear rate of the flow, as shown above, while having the advantage of allowing a direct comparison of the true shear stresses for both blood analogues.

For the upper and lower walls, the main difference relative to the Newtonian case is found at the inlet branch and differences can be as large as $30 \%$ on account of the lower viscosities due to the shear-thinning nature of the non-Newtonian fluid. Apart from this the non-Newtonian stress profiles along the main duct faithfully follow the corresponding Newtonian profiles and on the main outlet duct the profiles tend to collapse suggesting that the average viscosity for the GNF fluid is very close to the Newtonian viscosity value. In the branch duct, however, the differences are larger not only in terms of the local stress values but also in the extent of the stress variations along the walls: the stress profile for the non-Newtonian fluid shows larger variations along the wall than the corresponding Newtonian profile, with stress values sometimes exceeding those of the Newtonian fluid. Given the relevance of wall shear stress profiles for hemodynamic diseases, the differences seen in Figure 12 are clear evidence to the need to model blood rheology as accurately as possible.

Finally, Figure 17 shows contour plots of the shear stress inside the flow, which can be directly compared with those for the Newtonian flow of Figure 13. At the four time moments shown, 
the stresses for the non-Newtonian flow are generally lower than those for the Newtonian blood analogue, but note that some correspond to different moments as far as the dynamics of the vortices are concerned (cf. Figure 16). In spite of this note of caution, by contrasting Figures 13 and 17 we see that stress concentration is alleviated for the GNF case and the regions of high shear stresses are smaller in size.

\section{CONCLUSIONS}

A finite volume methodology, implementing at least second-order accurate methods in space and time, is used to investigate in detail steady and unsteady flows in a two-dimensional T-junction in the laminar regime for Newtonian and a non-Newtonian Carreau fluid. The flow conditions investigated are of relevance to hemodynamic applications and the main objective here was to quantify the accuracy of the predictions and to provide benchmark quality data prior to a more in-depth research programme with non-Newtonian inelastic and viscoelastic fluids.

For the steady flow, calculations were performed for Reynolds numbers varying between 50 and 1000 and flow rate ratios in the range of 0.1-0.9. The two recirculation lengths were seen to increase with Reynolds number, but regarding the effect of the flow rate ratio the behaviour was non-monotonic. For values of $\beta$ below 0.5 both recirculations increased followed by a decrease with further increases in $\beta$. Vortex data useful for benchmarking are given under tabulated form.

For the pulsating Newtonian flow in the T-junction, the investigation was carried out for an extraction ratio of $\beta=0.7$, a Reynolds number of 102 and a Womersley number of $\alpha=4.864$. Although the main recirculation in the side branch was always present, there is an instability that breaks the main vortex into two pieces at $t / T_{\mathrm{O}} \approx 0.81$ and convects part of the vortex structure downstream. The secondary vortex was found to be absent for a sixth of the time, for this value of $\beta$. The shear stress on the walls was also quantified and found to reach maxima at the walls opposite the recirculations, where the main stream impinges the wall. Very good match between our predictions and the experimental data of Khodadadi et al. [31] was achieved for local quantities such as velocity and shear stresses, along a time period, thus giving support to the quality of that measured data set, which has been scarcely used.

The main features of the pulsating flow for a non-Newtonian Carreau fluid analogue to blood are similar to those of the Newtonian analogue, with both recirculations being about $10 \%$ longer and also short lived than the corresponding Newtonian eddies. A phenomenon similar to that found for the Newtonian case is also present, with the main vortex in the branch breaking into two structures at $\frac{3}{4}$ time along the period, and the smaller structure being advected by the flow along the branch. However, the stresses for the non-Newtonian fluid are generally lower than the Newtonian stresses and by a large amount that can be as large as 30\% at the walls of the separated flow regions. Given the important relationship between wall stresses and hemodynamic diseases, these differences have implications and are a clear evidence to the need for properly representing the true rheology of blood if accurate predictions and investigations of blood flows are to be carried out.

Furthermore, the new finding related to the division of the main recirculating region at about $\frac{3}{4}$ of the pulsating period, with subsequent dragging of that portion of the vortex by the flow through the branch may be connected with some coronary diseases resulting from clotting of small vessels with lipids and other solid-like material that tend to accumulate in such recirculations. 


\section{APPENDIX A: ANALYTICAL SOLUTION FOR PULSATING LAMINAR CHANNEL FLOW OF NEWTONIAN FLUIDS}

The analytical solution for pulsating laminar channel flow of Newtonian fluids is presented in some classical books $[49,50]$. It is summarized here because it is an essential ingredient to the time-dependent validations of Section 3.2 and, in addition, it serves as inlet boundary condition in Sections 3.3 and 3.4. The channel in question coincides with the inlet plane to the T-junction (plane 1, see Figure 1), having a half-height $h=H / 2$, with the origin of the $y$ axis at the symmetry plane and streamwise coordinate $x$.

The flow is assumed to develop instantly leading to the following linear momentum equation (quasi-steady approximation):

$$
\frac{\partial u}{\partial t}=v \frac{\partial^{2} u}{\partial y^{2}}-\frac{1}{\rho} \frac{\mathrm{d} p}{\mathrm{~d} x}
$$

where the pressure gradient follows the sinusoidal variation of Equation (13) and with the no-slip boundary condition imposed at the walls $(y= \pm h)$. Since Equation (A1) is linear, the solution can be decomposed into the sum of steady $\left(u_{s}\right)$ and oscillating $\left(u_{\mathrm{O}}\right)$ contributions:

$$
u(y, t)=u_{s}(y)+u_{\mathrm{O}}(y, t)
$$

The steady velocity profile is the classical parabolic expression, here expressed in normalized form by adopting as velocity scale $K_{\mathrm{O}} / \omega$ and introducing the Womersley number $(\alpha=h / \sqrt{v / \omega})$ [50]:

$$
U_{s}(Y) \equiv \frac{u_{s}(y)}{K_{\mathrm{O}} / \omega}=\frac{\alpha^{2}}{2} \frac{K_{s}}{K_{\mathrm{O}}}\left[1-Y^{2}\right]
$$

where $Y=y / h$. To the constant part of the pressure gradient $\rho K_{s}$ it corresponds a steady bulk velocity, $\bar{u}_{s}=h^{2} K_{s} / 3 v$, which is identical to the average inlet velocity of the main text $\bar{u}_{1}$.

The analytical solution for the oscillating contribution is more difficult to derive, and is given by (see Reference [50]):

$$
U_{0}(Y, T) \equiv \frac{u_{\mathrm{O}}(y, t)}{K_{\mathrm{O}} / \omega}=\left[1-\frac{M(Y, \xi)}{J(\xi)}\right] \sin (T)-\frac{N(Y, \xi)}{J(\xi)} \cos (T)
$$

with $\xi=\alpha / \sqrt{2}, T=\omega t$, and the definitions:

$$
\begin{gathered}
J(\xi)=C^{2}(\xi)+S^{2}(\xi), \quad C(x)=\cosh (x) \cos (x), \quad S(x)=\sinh (x) \sin (x) \\
M(Y, \xi)=C(\xi Y) C(\xi)+S(\xi Y) S(\xi), \quad N(Y, \xi)=C(\xi Y) S(\xi)+S(\xi Y) C(\xi)
\end{gathered}
$$

While the above results for the velocity profile of pulsating channel flow can be found in textbooks, we needed further information such as shear stress and average velocity variations, which was derived as part of the present work. The shear stress is obtained by differentiation of 
the velocity profile $\left(\tau_{x y}=\eta \partial u / \partial y\right)$ and was found to be

$$
\begin{aligned}
\frac{\tau_{x y}}{\eta K_{\mathrm{O}} /(\omega h)}= & -\frac{\xi}{J(\xi)}\{[(A-B) C(\xi)+(A+B) S(\xi)] \sin (T) \\
& +[(A-B) S(\xi)-(A+B) C(\xi)] \cos (T)\}-\alpha^{2} \frac{K_{s}}{K_{\mathrm{O}}} Y
\end{aligned}
$$

where $A=\sinh (\xi Y) \cos (\xi Y)$ and $B=\cosh (\xi Y) \sin (\xi Y)$.

The centreline velocity $\left(u_{c}\right)$ is the maximum instantaneous velocity at each time and is obtained by setting $y=0$ in Equations (A3) and (A4). The phase angle of $u_{c}$ relative to the pressure gradient was calculated and is given by

$$
\delta=\arctan \left(-\frac{J(\xi)-M(0, \xi)}{N(0, \xi)}\right)
$$

This angle approaches $90^{\circ}$ when $N(0, \xi) \rightarrow 0$, which is the case for large Womersley numbers $(\alpha \geqslant 2)$. Another quantity of interest is the normalized instantaneous bulk velocity, obtained by integration of the velocity profile:

$$
\bar{u}(t) \equiv \int_{0}^{h} u(y, t) \mathrm{d} y=\frac{K_{\mathrm{O}}}{\omega}\left[\sin (T)-\frac{\Omega \sin (T)+\Lambda \cos (T)}{2 J(\xi)}\right]
$$

where

$$
\begin{aligned}
& \Omega=\exp (\xi)[P \sin (\xi)+Q \cos (\xi)]+\exp (-\xi)[P \sin (\xi)-Q \cos (\xi)] \\
& \Lambda=\exp (\xi)[P \cos (\xi)-Q \sin (\xi)]-\exp (-\xi)[P \cos (\xi)+Q \sin (\xi)] \\
& P=\frac{C(\xi)+S(\xi)}{2 \xi} \text { and } Q=\frac{C(\xi)-S(\xi)}{2 \xi}
\end{aligned}
$$

The phase angle between the bulk velocity and the pressure gradient can then be obtained by calculating the angle between the two corresponding complex quantities, and is given by

$$
\delta^{*}=\arctan \left(\frac{2 J(\xi)-\Omega}{\Lambda}\right)
$$

The velocity profile of Equation (A2), together with Equations (A3) and (A4), and the shear stress and average velocity profiles given by Equations (A5) and (A7), respectively, will be used in the main text.

\section{ACKNOWLEDGEMENTS}

The authors acknowledge the grants PBICT/C/QUI/1980/95 and POCTI/EME/48665/2002 from Fundação para a Ciência e Tecnologia (FCT, Portugal) and FEDER, and Professor Khodadadi for providing the raw data of Khodadadi et al. [31]. 


\section{REFERENCES}

1. Ku DN. Blood flow in arteries. Annual Review of Fluid Mechanics 1997; 29:399-434.

2. Berger SA, Jou L-D. Flows in stenotic vessels. Annual Review Fluid Mechanics 2000; 32:347-382.

3. Taylor CA, Draney MT. Experimental and computational methods in cardiovascular fluid mechanics. Annual Review of Fluid Mechanics 2004; 36:197-231.

4. Popel AS, Johnson PC. Microcirculation and hemorheology. Annual Review of Fluid Mechanics 2005; 37:43-69.

5. Deutsch S, Tarbell JM, Manning KB, Rosenberg G, Fontaine AA. Experimental fluid mechanics of pulsatile artificial blood pumps. Annual Review of Fluid Mechanics 2006; 38:65-86.

6. Thilmany J. Ask the supercomputer. Mechanical Engineering 2006; 128:36-38.

7. Fung YC. Biomechanics: Circulation. Springer: Heidelberg, 1997.

8. Pedley TJ. The Fluid Mechanics of Large Blood Vessels. Cambridge University Press: Cambridge, 1980.

9. Ravensbergen J, Krijger JKB, Hillen B, Hoogstraten HW. Merging flows in an arterial confluence: the vertebrobasilar junction. Journal of Fluid Mechanics 1995; 304:119-141.

10. Balducci A, Grigioni M, Querzoli G, Romano GP, Daniele C, D’Avenio G, Barbaro V. Investigation of the flow field downstream of an artificial heart valve by means of PIV and PTV. Experiments in Fluids 2004; 36:204-213.

11. Cenedese A, Del Prete Z, Miozzo M, Querzoli G. A laboratory investigation of the flow in the left ventricle of a human heart with prosthetic, tilting-disk valves. Experiments in Fluids 2005; 39:322-335.

12. Ghia U, Ghia KN, Shin CT. High-Re solutions for incompressible flow using the Navier-Stokes equations and a multigrid method. Journal of Computational Physics 1982; 48:387-411.

13. Brown RA, McKinley GH. Report on the VIII International Workshop on Numerical Methods in Viscoelastic Flows. Journal of Non-Newtonian Fluid Mechanics 1994; 52:407-413.

14. Alves MA, Oliveira PJ, Pinho FT. Benchmark solutions for the flow of Oldroyd-B and PTT fluids in planar contractions. Journal of Non-Newtonian Fluid Mechanics 2003; 110:45-75.

15. Mazumdar JN. Biofluid Mechanics. World Scientific: Singapore, 1992.

16. Owens RG. A new microstructure-based constitutive model for human blood. Journal of Non-Newtonian Fluid Mechanics 2006; 140:57-70.

17. Thurston GB. Rheological parameters for the viscosity, viscoelasticity and thixotropy of blood. Biorheology 1979; 16:149-162.

18. Picart C, Piau JM, Galliard H, Carpenter P. Human blood yield stress and its hematocrit dependence. Journal of Rheology 1998; 42:1-12.

19. Vlastos G, Lerche D, Koch B, Samba O, Pohl M. The effect of parallel combined steady and oscillatory shear flows on blood and polymer solutions. Rheologica Acta 1997; 36:160-172.

20. Wells RE, Merrill EW, Gabelnick H. Shear rate dependence of viscosity of blood interaction of red cells and plasma proteins. Transactions of the Society of Rheology 1962; 6:19-24.

21. Perktold K, Resch M, Florian H. Pulsatile non-Newtonian flow characteristics in a three-dimensional human carotid bifurcation model. ASME Journal of Biomechanical Engineering 1991; 113:464-475.

22. Perktold K, Peter RO, Resch M, Langs G. Pulsatile non-Newtonian flow in three-dimensional carotid bifurcation models: a numerical study of flow phenomena under different bifurcation angles. Journal of Biomechanical Engineering (ASME) 1991; 113:507-515.

23. Ballyk PD, Steinman DA, Ethier CR. Simulation of non-Newtonian blood flow in an end-to-side anastomosis. Biorheology 1994 31:565-586.

24. Bachmann C, Hugo G, Rosenberg G, Deutsch S, Fontainee AA, Tarbell JM. Fluid dynamics of a pediatric ventricular assist device. Artificial Organs 2000; 24:362-372 [see also Erratum in Artificial Organs 2000; 24: 989].

25. Gray JD, Owen I, Escudier MP. Dynamic scaling of unsteady shear-thinning non-Newtonian fluid flows in a large-scale model of a distal anastomosis. Experiments in Fluids 2007; 43:535-546.

26. Vogel G. Investigation of the loss in right-angled pipe branches. Mitt. Hydraulischen Instituts der Tech. Hochschule München 1926; 1:75-90 (Translated by Voetsch C, Technical Memorandum n. 299, US Bureau of Reclamation, 1932).

27. Costa NP, Maia R, Pinho FT, Proença MF. Edge effects on the flow characteristics in a $90^{\circ}$ tee junction. Journal of Fluids Engineering (ASME) 2006; 128(6):1204-1217.

28. Karino T, Kwong HHM, Goldsmith HL. Particle flow behaviour in models of branching vessels. I-Vortices in 90 T-junctions. Biorheology 1979; 16:231-248.

29. Liepsch D, Moravec S, Rastogi AK, Vlachos NS. Measurement and calculations of laminar flow in a ninety degree bifurcation. Journal of Biomechanics 1982; 15:473-485. 
30. Khodadadi JM, Nguyen TM, Vlachos NS. Laminar forced convective heat transfer in a two-dimensional $90^{\circ}$ bifurcation. Numerical Heat Transfer 1986; 9:677-695.

31. Khodadadi JM, Vlachos NS, Liepsch D, Moravec S. LDA measurements and numerical prediction of pulsatile laminar flow in a plane 90-degree bifurcation. Journal of Biomechanical Engineering 1988; 110:129-136.

32. Khodadadi JM. Wall pressure and shear stress variations in a 90-deg bifurcation during pulsatile laminar flow. Journal of Fluids Engineering 1991; 113:111-115.

33. Moravec S, Liepsch D. Flow investigations in a model of a three-dimensional human artery with Newtonian and non-Newtonian fluids. Biorheology 1983; 20:745-759.

34. Liepsch D, Moravec S. Pulsatile flow of non-Newtonian fluid in distensible models of human arteries. Biorheology 1984; 21:571-586.

35. Rindt CCM, Steenhoven AAV. Modelling flow properties in biofluids. Journal of Biomechanical Engineering 1996; 118:90-96.

36. Oliveira PJ, Pinho FT, Pinto GA. Numerical simulation of non-linear elastic flows with a general collocated finite-volume method. Journal of Non-Newtonian Fluid Mechanics 1998; 79:1-43.

37. Oliveira PJ. Method for time-dependent simulations of viscoelastic flows: vortex shedding behind cylinder. Journal of Non-Newtonian Fluid Mechanics 2001; 101:113-137.

38. Alves MA, Oliveira PJ, Pinho FT. A convergent and universally bounded interpolation scheme for the treatment of advection. International Journal for Numerical Methods in Fluids 2003; 41:47-75.

39. Roache PJ. Quantification of uncertainty in computational fluid dynamics. Annual Review of Fluid Mechanics 1997; 29:123-160.

40. Escudier MP, Gouldson IW, Oliveira PJ, Pinho FT. Effects of inner cylinder rotation on laminar flow of a Newtonian fluid through an eccentric annulus. International Journal of Heat and Fluid Flow 2000; 21:92-103.

41. Escudier MP, Oliveira PJ, Pinho FT, Smith S. Fully-developed laminar flow of non-Newtonian liquids through annuli: comparison of numerical calculations with experiments. Experiments in Fluids 2002; 33:101-111.

42. Vlachos NS. Laminar flow through a plane $90^{\circ}$ bifurcation. Technical Report, Imperial College of Science and Technology, London, U.K., 1978.

43. Hayes RE, Nandakumar K, Nasr-El-Din H. Steady laminar flow in a 90 degree planar branch. Computers and Fluids 1989; 17:537-553.

44. Collins MW, Xu XY. A predictive scheme for flow in arterial bifurcations: comparison with laboratory measurements. Biomechanical Transport Processes. Plenum Press: New York, 1990; 125-133.

45. Neary VS, Sotiropoulos F. Numerical investigation of laminar flows through 90-degree diversion of rectangular cross-section. Computers and Fluids 1996; 25:95-118.

46. Shah RK, London AL. Laminar Flow Forced Convection in Ducts. Academic Press: New York, 1978.

47. Gaskell PH, Lau AKC. Curvature compensated convective transport: SMART, a new boundedness preserving transport algorithm. International Journal for Numerical Methods in Fluids 1988; 8:617-641.

48. Song B, Liu GR, Lam KY, Amano RS. On a higher-order discretisation scheme. International Journal for Numerical Methods in Fluids 2000; 32:881-897.

49. Schlichting H, Gersten K. Boundary-Layer Theory (8th edn). Springer: Berlin, 2000; 137.

50. Landau LD, Lifshitz EM. Fluid Mechanics. Pergamon Press, Addison-Wesley: London, Reading, MA, 1959. Translation from Russian edition of 1953; 95.

51. Caro CG, Fitz-Gerald JM, Schroter RC. Atheroma and arterial wall shear. Proceedings of the Royal Society of London B 1971; 177:109-159.

52. Blackshear PL. Mechanical haemolysis in flowing blood. Biomechanics_Its Foundations and Objectives, Fung YC, Perrone N, Anliker M (eds). Prentice-Hall, Inc.: Englewood Cliffs, NJ, U.S.A., 1972; 501-528.

53. Champion JV, North PF, Coakley WT, Williams AR. Shear fragility of human erythrocytes. Biorheology 1971; 8:23-29.

54. Banerjee RK, Cho YI, Kensey KR. A study of local hydrodynamics in a $90^{\circ}$ branched vessel with extreme pulsatile flows. International Journal of Computational Fluid Dynamics 1998; 9:23-42.

55. Cho YI, Kensey KR. Effects of the non-Newtonian viscosity of blood on flows in a diseased arterial vessel. Part 1: Steady flows. Biorheology 1991; 28:241-262. 